\title{
Comparison of Ru- and Mo-Based Chiral Olefin Metathesis Catalysts. Complementarity in Asymmetric Ring-Opening/Cross-Metathesis Reactions of Oxa- and Azabicycles
}

\author{
G. Alex Cortez, ${ }^{\dagger}$ Carl A. Baxter, ${ }^{\dagger}$ Richard R. Schrock, ${ }^{\#}$ and Amir H. Hoveyda, ${ }^{*}$, \\ ${ }^{\dagger}$ Department of Chemistry, Merkert Chemistry Center, Boston College \\ Chestnut Hill, Massachusetts 02467 \\ \#Department of Chemistry, Massachusetts Institute of Technology \\ Cambridge, Massachusetts 02139
}

\section{SUPPORTING INFORMATION}

General: All reactions were conducted in oven- $\left(135^{\circ} \mathrm{C}\right)$ or flame-dried glassware under an inert atmosphere of dry $\mathrm{N}_{2}$, unless otherwise stated. Infrared (IR) spectra were recorded on a Nicolet 210 spectrophotometer, $v_{\max }$ in $\mathrm{cm}^{-1}$. Bands are characterized as broad (br), strong (s), medium (m) or weak (w). ${ }^{1} \mathrm{H}$ NMR spectra were recorded on a Varian Gemini $2000(400 \mathrm{MHz})$ spectrometer. Chemical shifts are reported in ppm from tetramethylsilane with the solvent resonance as the internal standard $\left(\mathrm{CDCl}_{3}: \delta 7.26\right.$, $\mathrm{C}_{6} \mathrm{D}_{6}: \delta$ 7.16). Data are reported as follows: chemical shift, multiplicity $(\mathrm{s}=$ singlet, $\mathrm{d}=$ doublet, $\mathrm{t}=$ triplet, $\mathrm{q}=$ quartet, $\mathrm{br}=$ broad, $\mathrm{m}=$ multiplet or combinations thereof), coupling constants $(\mathrm{Hz})$, and integration. ${ }^{13} \mathrm{C}$ NMR spectra were recorded on a Varian Gemini $2000(100 \mathrm{MHz})$ spectrometer with complete proton decoupling. Chemical shifts are reported in ppm from tetramethylsilane with the solvent resonance as the internal reference $\left(\mathrm{CDCl}_{3}: \delta 77.16, \mathrm{C}_{6} \mathrm{D}_{6}: \delta 128.10\right)$. Enantiomer ratios were determined by chiral HPLC (Chiral Technologies Chiralpak OD column, Chiralpak AD, Chiralcel OJ, and Chiralcel OB-H $(4.6 \mathrm{~mm} \times 250 \mathrm{~mm})$ ). High-resolution mass spectrometry was performed at the University of Illinois Mass Spectrometry Laboratories (UrbanaChampaign, IL). Enantiomeric rotation values were recorded on a Rudolph Research Analytical Autopol IV polarimeter. Melting points were measured on a Thomas Hoover capillary melting point apparatus and are uncorrected. The absolute stereochemistry of piperidine products was assigned by inference based on the absolute stereochemistry of a previously reported piperidine product. ${ }^{1}$

Materials: All reagent solvents were purchased from Doe and Ingalls. Solvents were purged with argon and then purified under a positive pressure of dry argon by a modified Innovative Technologies purification system: $\mathrm{CH}_{2} \mathrm{Cl}_{2}$ was passed through activated

(1) See supporting information in: Cortez, G. A.; Schrock, R. R.; Hoveyda, A. H. Angew. Chem., Int. Ed. 2007, 119, 4618-1622. 
alumina columns; benzene was passed successively through activated $\mathrm{Cu}$ and alumina columns. Tetrahydrofuran was purified by distillation from sodium benzophenone ketal immediately prior to use. All reagents were purchased from Aldrich Chemical Company, Lancaster Synthesis, or Strem Chemicals, Inc., and purified by appropriate methods prior to use. Lithium aluminum hydride was purchased in powder form and was stored in a $\mathrm{N}_{2}$-filled glovebox. Mo complex 6 was prepared according to published procedures. ${ }^{2} \mathrm{Mo}$ complexes were handled under an inert atmosphere in a $\mathrm{N}_{2}$-filled glovebox. Substrates employed in Mo-catalyzed reactions were dried by repeated (three times) azeotropic distillation of water with benzene under high vacuum unless otherwise stated. $\mathrm{Ru}$ complexes 1a-2b were prepared according to published procedures. ${ }^{3}$ All reactions were allowed to stir with a magnetic stir bar and performed at $22{ }^{\circ} \mathrm{C}$, unless otherwise stated. All filtrations involved gravity filtration conducted with Whatman ${ }^{\circledR}$ filter papers.

Representative procedure for Mo-catalyzed asymmetric ring-opening metathesis/cross-metathesis reactions: ${ }^{4}$ In a $\mathrm{N}_{2}$-filled glovebox, Mo complex 6 (2.4 $\mathrm{mg}, 0.0030 \mathrm{mmol})$ was dissolved in $\mathrm{C}_{6} \mathrm{H}_{6}(0.250 \mathrm{~mL})$ in a $4-\mathrm{mL}$ vial. Styrene $(68.0 \mu \mathrm{L}$, $0.593 \mathrm{mmol})$ was added to this solution by syringe. ${ }^{5}$ The resulting mixture was allowed to stir for $1 \mathrm{~min}$ and then added by syringe to a solution of azabicycle 18 (15.0 $\mathrm{mg}$, $0.0592 \mathrm{mmol})$ in $\mathrm{C}_{6} \mathrm{H}_{6}(0.250 \mathrm{~mL})$ in a $4-\mathrm{mL}$ vial. The mixture was allowed to stir for 1 h. At this time, the vial was removed from the glovebox, and the volatiles were removed in vacuo. The resulting dark brown residue was purified by silica gel chromatography (9:1 $\left.\mathrm{CH}_{2} \mathrm{Cl}_{2}: \mathrm{MeOH}\right)$ to afford piperidine 19 as colorless oil $(20.4 \mathrm{mg}, 0.0570 \mathrm{mmol}$, 95\%). IR (neat): 2949 (s), 2930 (s), 2772 (s), 2363 (s), 1646 (w). ${ }^{1} \mathrm{H}$ NMR (400 MHz, $\left.\mathrm{CDCl}_{3}\right): \delta 7.39-7.20(\mathrm{~m}, 5 \mathrm{H}), 6.48(\mathrm{~d}, J=15.6 \mathrm{~Hz}, 1 \mathrm{H}), 6.15(\mathrm{dd}, J=15.6,8.8 \mathrm{~Hz}, 1 \mathrm{H})$, 5.79 (ddd, $J=17.6,10.4,8.8 \mathrm{~Hz}, 1 \mathrm{H}), 5.15(\mathrm{dd}, J=17.2,1.6 \mathrm{~Hz}, 1 \mathrm{H}), 5.05(\mathrm{dd}, J=10.4$, $1.6 \mathrm{~Hz}, 1 \mathrm{H}), 3.77-3.68(\mathrm{~m}, 1 \mathrm{H}), 2.77-2.60(\mathrm{~m}, 1 \mathrm{H}), 2.58-2.48(\mathrm{~m}, 1 \mathrm{H}), 2.18(\mathrm{~s}, 3 \mathrm{H})$, $1.87-1.78(\mathrm{~m}, 2 \mathrm{H}), 1.60-1.51(\mathrm{~m}, 2 \mathrm{H}), 0.87(\mathrm{~s}, 9 \mathrm{H}), 0.05(\mathrm{~s}, 6 \mathrm{H}) .{ }^{13} \mathrm{C}$ NMR $(100 \mathrm{MHz}$, $\left.\mathrm{CDCl}_{3}\right): \delta 141.9,137.1,133.4,130.7,128.7,127.6,126.4,115.7,68.7,67.6,66.7,43.0$, 42.9, 41.5, 25.9, 18.3, -4.39. HRMS EI $(\mathrm{m} / \mathrm{z})$ Calcd for $\mathrm{C}_{22} \mathrm{H}_{35} \mathrm{NOSi} 357.2488(\mathrm{M})^{+}$, Found 357.2482.

(2) Tsang, W. C. P.; Jernelius, J. A.; Cortez, G. A.; Weatherhead, G. S.; Schrock, R. R.; Hoveyda, A. H. J. Am. Chem. Soc. 2003, 2591-2596. For the synthesis of other chiral Mo complexes, see: Schrock R. R.; Hoveyda, A. H. Angew. Chem. Int. Ed. 2003, 42, 4558-4565.

(3) a) Van Veldhuizen, J. J.; Garber, S. B.; Kingsbury, J. S.; Hoveyda, A. H. J. Am. Chem. Soc. 2002, 124, 4954-4955. b) Van Veldhuizen, J. J.; Campbell, J. E.; Giudici, R. E.; Hoveyda, A. H. J. Am. Chem. Soc. 2005, 127, 6877-6882.

(4) The Characterization date for piperidines 19 and 19-OH was previously reported, see: Cortez, G. A.;

Schrock, R. R.; Hoveyda, A. H. Angew. Chem., Int. Ed. 2007, 119, 4618-1622.

(5) Mo complex 6 was pre-treated with styrene to ensure formation of the chiral Mo benzylidene complex. 
- Representative procedure for removal of silyl protecting group in piperidine products: ${ }^{3,6}$ A 4-mL vial was charged with 19 (20.4 mg, $\left.0.0570 \mathrm{mmol}\right)$, THF (0.500 $\mathrm{mL})$, and TBAF (285 $\mu \mathrm{L}, 0.285 \mathrm{mmol}$, a $1.00 \mathrm{M}$ solution in THF). The vial was tightly sealed with a Teflon cap, placed in a heating mantle at $65{ }^{\circ} \mathrm{C}$, and the mixture was allowed to stir for $1 \mathrm{~h}$. At this time, the vial was allowed to cool to $22{ }^{\circ} \mathrm{C}$ over a period of $15 \mathrm{~min}$, and the volatiles were removed in vacuo. The resulting yellow residue was purified by silica gel chromatography $\left(4: 1 \mathrm{CH}_{2} \mathrm{Cl}_{2}: \mathrm{MeOH}\right)$ to afford piperidine 19-OH as colorless oil (13.2 mg, $0.0541 \mathrm{mmol}, 95 \%)$. ${ }^{1} \mathrm{H} \mathrm{NMR}\left(400 \mathrm{MHz}, \mathrm{CDCl}_{3}\right): \delta 7.38-7.21$ $(\mathrm{m}, 5 \mathrm{H}), 6.49(\mathrm{~d}, J=15.6 \mathrm{~Hz}, 1 \mathrm{H}), 6.16(\mathrm{dd}, J=15.6,8.8 \mathrm{~Hz}, 1 \mathrm{H}), 5.76(\mathrm{ddd}, J=17.6$, $10.4,8.8 \mathrm{~Hz}, 1 \mathrm{H}), 5.17(\mathrm{dd}, J=17.2,1.6 \mathrm{~Hz}, 1 \mathrm{H}), 5.07$ (dd, $J=10.4,1.6 \mathrm{~Hz}, 1 \mathrm{H})$, 3.82-3.70 (m, 1H), 2.77-2.60 (m, 1H), 2.58-2.48 (m, 1H), $2.20(\mathrm{~s}, 3 \mathrm{H}), 2.00-1.92(\mathrm{~m}$, $2 \mathrm{H}), 1.55-1.43(\mathrm{~m}, 3 \mathrm{H}) . \quad[\alpha]_{\mathrm{D}}{ }^{20}-75.2\left(c=0.2, \mathrm{CHCl}_{3}\right)$ for a sample of $94 \% e e$. The enantiomeric purity of this compound was determined by HPLC analysis in comparison with authentic racemic material, shown below: Chiralpak OD (4.6 x $250 \mathrm{~mm})$, 99:1 hexanes: $i-\mathrm{PrOH}, 1.0 \mathrm{~mL} / \mathrm{min}, \lambda=254 \mathrm{~nm}, 94 \%$ ee.

\section{Authentic racemic}

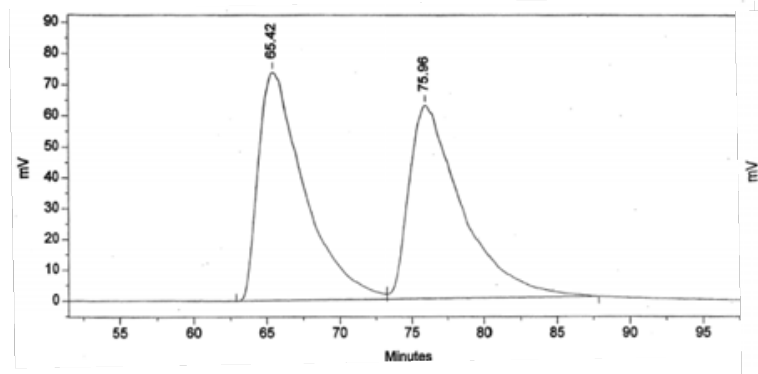

94\% ee with Mo catalyst 6
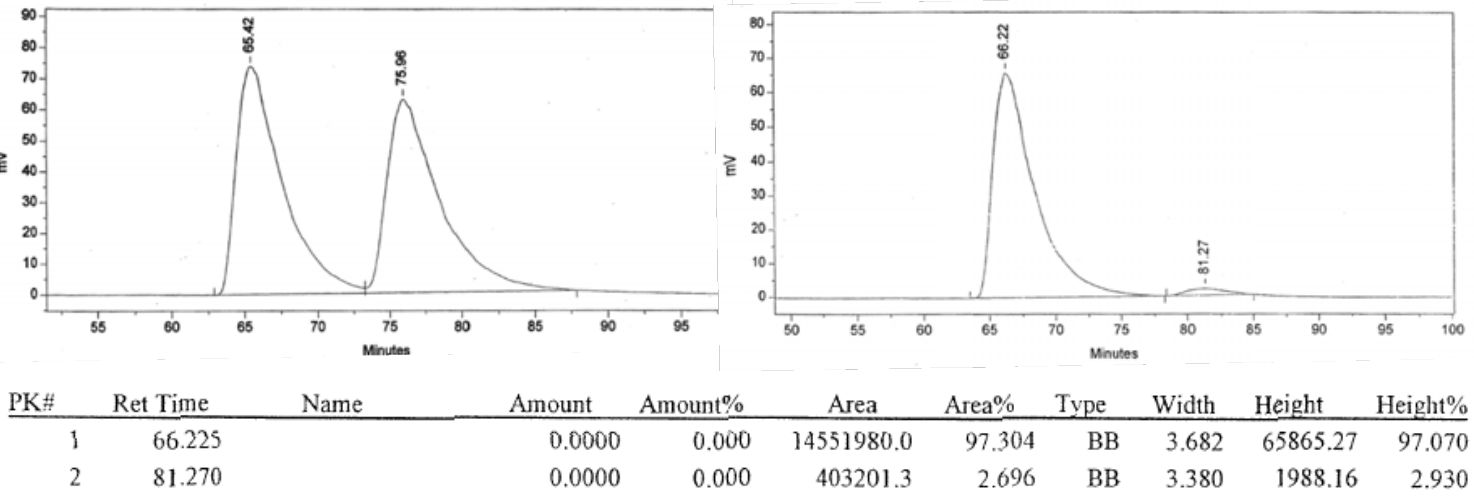

\begin{tabular}{rrrrrrr} 
t\% & \multicolumn{1}{c}{ Area } & \multicolumn{1}{c}{ Area\% } & Type & Width & \multicolumn{1}{c}{ Height } & Height\% \\
\hline 000 & 14551980.0 & 97.304 & BB & 3.682 & 65865.27 & 97.070 \\
.000 & 403201.3 & 2.696 & BB & 3.380 & 1988.16 & 2.930
\end{tabular}

Representative procedure for Ru-catalyzed asymmetric ring-opening metathesis/cross-metathesis reactions. Method A: $\mathrm{Ru}$ complex $1 \mathrm{a}$ (3.8 mg, 0.0046 mmol) was dissolved in styrene $(106 \mu \mathrm{L}, 0.930 \mathrm{mmol})$ in a $4-\mathrm{mL}$ vial. The resulting mixture was added by syringe to a solution of azabicycle $20(20.0 \mathrm{mg}, 0.0930 \mathrm{mmol})$ in styrene $(106 \mu \mathrm{L}, 0.930 \mathrm{mmol})$ in a $4-\mathrm{mL}$ vial. The mixture was allowed to stir for $24 \mathrm{~h}$, upon which time the volatiles were removed in vacuo. The resulting dark brown residue was purified by silica gel column chromatography $\left(98: 2 \quad \mathrm{CH}_{2} \mathrm{Cl}_{2}: \mathrm{MeOH}\right)$ to give piperidine 21 as colorless oil (23.8 mg, $0.0744 \mathrm{mmol}, 80 \%$ ). IR (neat): 3363 (s), 2955 (m), 2930 (m), 2338 (s), 1104 (s). ${ }^{1} \mathrm{H}$ NMR (400 MHz, $\left.\mathrm{CDCl}_{3}\right): \delta 7.29-7.12(\mathrm{~m}, 10 \mathrm{H})$, $6.44(\mathrm{~d}, J=15.9 \mathrm{~Hz}, 1 \mathrm{H}), 5.99$ (dd, $J=15.9,8.6 \mathrm{~Hz}, 1 \mathrm{H}), 5.81$ (ddd, $J=17.6,10.0,8.8$ $\mathrm{Hz}, 1 \mathrm{H}), 5.18(\mathrm{dd}, J=17.2,0.9 \mathrm{~Hz}, 1 \mathrm{H}), 5.04(\mathrm{dd}, J=10.2,1.5 \mathrm{~Hz}, 1 \mathrm{H}), 3.92$ (d, $J=15.3$ $\mathrm{Hz}, 1 \mathrm{H}), 3.73$ (d, $J=15.3 \mathrm{~Hz}, 1 \mathrm{H}), 3.69-3.64$ (m, 1H), 3.07 (ddd, $J=14.4,9.2,2.8 \mathrm{~Hz}$,

(6) Determination of enantiomeric excess of 19 involved the piperidine derived from deprotection of the secondary alcohol in $19\left((n-\mathrm{Bu})_{4} \mathrm{NF}\right.$ in THF, $\left.22{ }^{\circ} \mathrm{C}\right)$. 
1H), 3.01 (ddd, $J=11.2,8.8,2.4 \mathrm{~Hz}, 1 \mathrm{H}), 1.80-1.76(\mathrm{~m}, 2 \mathrm{H}), 1.62-1.51(\mathrm{~m}, 2 \mathrm{H}), 0.86$ (s, 9H), 0.04 (s, 6H). ${ }^{13} \mathrm{C}$ NMR (100 MHz, $\left.\mathrm{CDCl}_{3}\right): \delta .142 .7,140.1,137.2,134.6,130.0$, 129.3, 128.5, 127.8, 127.3, 126.3, 126.2, 115.6, 68.6, 64.4, 64.0, 55.2, 43.4, 43.4, 25.9, 18.2, -4.5. HRMS EI $(\mathrm{m} / \mathrm{z})$ mass Calcd for $\mathrm{C}_{28} \mathrm{H}_{40} \mathrm{NOSi} 434.2879(\mathrm{M}+\mathrm{H})^{+}$, Found 434.2889. $[\alpha]_{\mathrm{D}}{ }^{20}-63.52\left(c=0.5, \mathrm{CHCl}_{3}\right)$ for a sample of $94 \%$ ee. Determination of enantiomeric excess of $\mathbf{2 1}$ involved piperidine 23a, derived from TBAF deprotection ${ }^{4}$ of the secondary alcohol in 21. Piperidine 23a. IR (neat): 3333 (br), 3024 (w), 2936 (m), 2911 (m), 1495 (m), 1457 (m). ${ }^{1} \mathrm{H}$ NMR (400 MHz, $\left.\mathrm{CDCl}_{3}, \mathrm{TMS}\right): \delta$ 7.30-7.09 (m, $10 \mathrm{H}), 6.45(\mathrm{~d}, J=15.9 \mathrm{~Hz}, 1 \mathrm{H}), 5.98(\mathrm{dd}, J=15.9,8.6 \mathrm{~Hz}, 1 \mathrm{H}), 5.82$ (ddd, $J=17.2$, $10.1,8.4 \mathrm{~Hz}, 1 \mathrm{H}), 5.21(\mathrm{dd}, J=17.2,1.5 \mathrm{~Hz}, 1 \mathrm{H}), 5.07(\mathrm{dd}, J=10.1,1.5 \mathrm{~Hz}, 1 \mathrm{H}), 3.94$ (d, $J=15.5 \mathrm{~Hz}, 1 \mathrm{H}), 3.74(\mathrm{~d}, J=15.5 \mathrm{~Hz}, 1 \mathrm{H}), 3.76-3.68(\mathrm{~m}, 1 \mathrm{H}), 3.11$ (ddd, $J=11.6$, 8.8, $2.8 \mathrm{~Hz}, 1 \mathrm{H}), 3.05$ (ddd, $J=11.2,8.4,2.8 \mathrm{~Hz}, 1 \mathrm{H}), 1.96-1.90$ (m, $2 \mathrm{H}), 1.64$ (br s, $1 \mathrm{H}), 1.58-1.48(\mathrm{~m}, 2 \mathrm{H}) .{ }^{13} \mathrm{C}$ NMR $\left(100 \mathrm{MHz}, \mathrm{CDCl}_{3}\right): \delta 142.4,140.1,137.1,134.2$, 130.2, 129.2, 128.5, 127.9, 127.4, 126.3, 126.2, 115.9, 68.0, 64.4, 64.1, 55.2, 43.0. HRMS EI $(\mathrm{m} / z)$ mass Calcd for $\mathrm{C}_{22} \mathrm{H}_{25} \mathrm{NO} 319.1936(\mathrm{M})^{+}$, Found 319.1938. The enantiomeric purity of this compound was determined by HPLC analysis in comparison with authentic racemic material, shown below: Chiralpak OD (4.6 x $250 \mathrm{~mm}), 98: 2$ hexanes: $i-\mathrm{PrOH}, 1.0 \mathrm{~mL} / \mathrm{min}, \lambda=254 \mathrm{~nm},>98 \%$ ee.

\section{Authentic racemic}
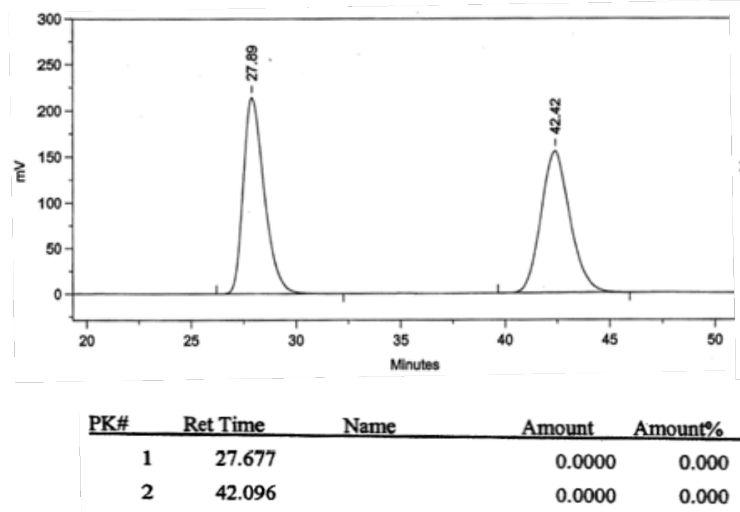

Authentic racemic

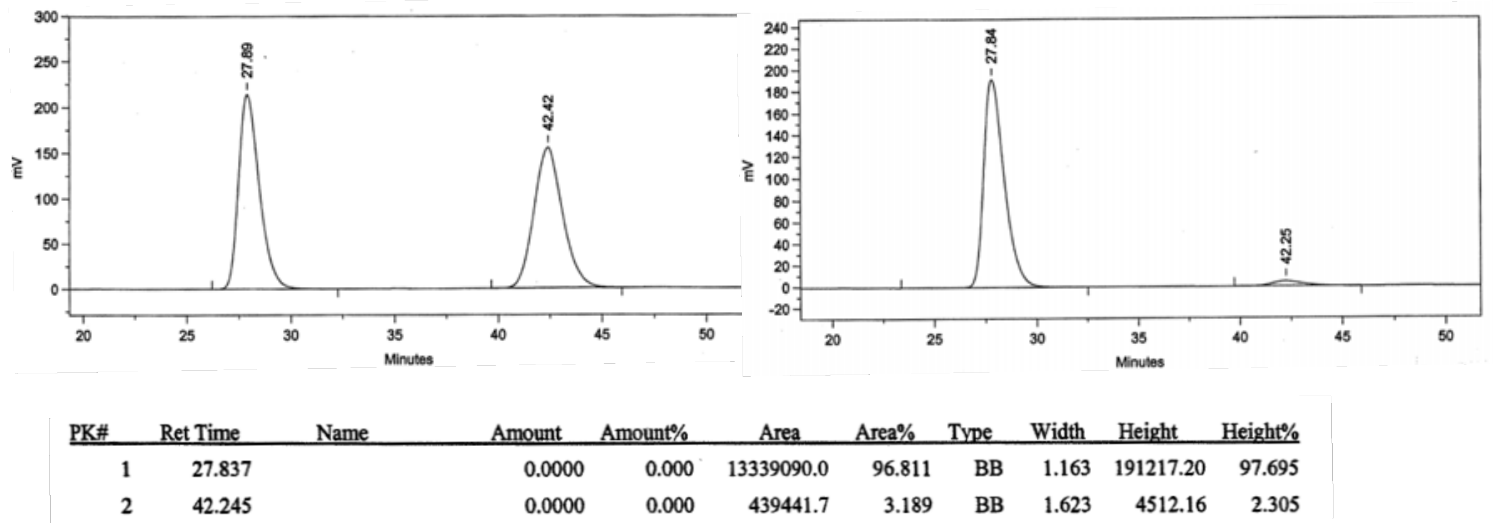

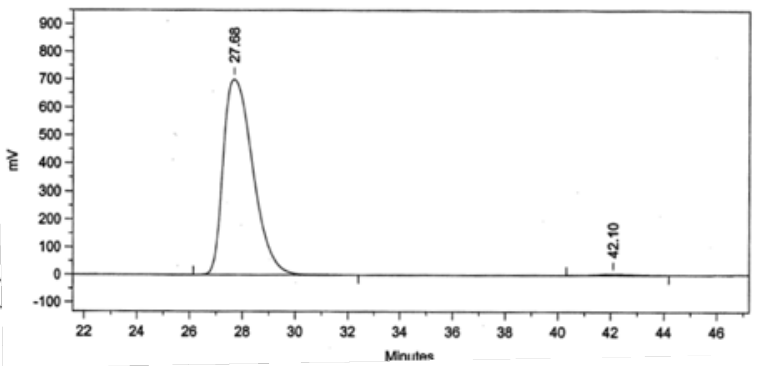

\begin{tabular}{rrrrrrr} 
nt\% & \multicolumn{1}{c}{ Area } & \multicolumn{1}{c}{ Area\% } & Type & Width & Height & Height\% \\
\hline 0.000 & 54585420.0 & 99.247 & BB & 1.300 & 699891.60 & 99.341 \\
0.000 & 414404.3 & 0.753 & BB & 1.489 & 4639.53 & 0.659
\end{tabular}

94\% ee with Ru catalyst 2a (entry 6 of Table 3) 
Representative procedure for Ru-catalyzed asymmetric ring-opening metathesis/cross-metathesis reactions. Method B for piperidine 23f: Ru complex 1a (2.8 $\mathrm{mg}, 0.0035 \mathrm{~mol})$ was dissolved in toluene $(37.0 \mu \mathrm{L}, 0.350 \mathrm{mmol})$ in a $4-\mathrm{mL}$ vial. The resulting mixture was added by syringe to a solution of azabicycle $22(15.0 \mathrm{mg}$, $0.0697 \mathrm{mmol})$, and $p$-trifluoromethylstyrene $(51.0 \mu \mathrm{L}, 0.350 \mathrm{mmol})$ in a $4-\mathrm{mL}$ vial. The resulting solution was allowed to stir for $12 \mathrm{~h}$, at which time another portion of $\mathrm{Ru}$ complex 1a $(2.8 \mathrm{mg}, 0.0035 \mathrm{mmol})$ was added. The resulting mixture was allowed to stir for a further $12 \mathrm{~h}$, upon which time the volatiles were removed in vacuo. The resulting dark brown residue was purified by silica gel column chromatography $\left(99: 1 \mathrm{CH}_{2} \mathrm{Cl}\right.$. 2: $\mathrm{MeOH})$ to furnish piperidine $\mathbf{2 3 f}$ as colorless oil $(21.0 \mathrm{mg}, 0.0540 \mathrm{mmol}, 78 \%)$. IR (neat): 3358 (br), 2936 (w), 2848 (w), 1608 (w), 1325 (s), 1155 (m), 1124 (m). ${ }^{1} \mathrm{H}$ NMR (400 MHz, CDCl, $\mathrm{TMS}): \delta 7.46$ (d, $J=8.3 \mathrm{~Hz}, 2 \mathrm{H}), 7.28-7.16(\mathrm{~m}, 5 \mathrm{H}), 7.10(\mathrm{~d}, J=8.3$ $\mathrm{Hz}, 2 \mathrm{H}), 6.44(\mathrm{~d}, J=16.1 \mathrm{~Hz}, 1 \mathrm{H}), 5.99$ (dd, $J=16.1,8.8 \mathrm{~Hz}, 1 \mathrm{H}), 5.83$ (ddd, $J=17.3$, $10.2,8.4 \mathrm{~Hz}, 1 \mathrm{H}), 5.21(\mathrm{dd}, J=17.3,0.6 \mathrm{~Hz}, 1 \mathrm{H}), 5.08(\mathrm{dd}, J=10.2,1.3 \mathrm{~Hz}, 1 \mathrm{H}), 3.98$ (d, $J=15.4 \mathrm{~Hz}, 1 \mathrm{H}), 3.75-3.70(\mathrm{~m}, 1 \mathrm{H}), 3.62(\mathrm{~d}, J=15.4 \mathrm{~Hz}, 1 \mathrm{H}), 3.11$ (ddd, $J=11.2$, 8.8, $2.6 \mathrm{~Hz}, 1 \mathrm{H}), 3.04(\mathrm{ddd}, J=11.2,8.4,2.6 \mathrm{~Hz}, 1 \mathrm{H}), 1.97-1.89(\mathrm{~m}, 2 \mathrm{H}), 1.57$ (br s, $1 \mathrm{H}), 1.59-1.49(\mathrm{~m}, 2 \mathrm{H}) .{ }^{13} \mathrm{C}$ NMR $\left(100 \mathrm{MHz}, \mathrm{CDCl}_{3}\right): \delta 142.2,140.6,140.4,137.1$, 129.0, 128.6, 128.0, 126.5, 126.3, 125.4, 125.3, 116.2, 68.0, 64.6, 64.6, 55.7, 42.9 42.7, -5.1. HRMS ES $(\mathrm{m} / \mathrm{z})$ mass Calcd for $\mathrm{C}_{23} \mathrm{H}_{25} \mathrm{~F}_{3} \mathrm{NO} 388.1888(\mathrm{M}+\mathrm{H})^{+}$, Found 388.1878. $[\alpha]_{\mathrm{D}}^{25}-72.9\left(c 0.5, \mathrm{CHCl}_{3}\right)$ for a sample of $97 \%$ ee. The enantiomeric purity of this compound was determined by HPLC analysis in comparison with authentic racemic material, shown below: Chiralpak OD $(4.6 \times 250 \mathrm{~mm}), 98: 2$ hexanes: $i-\mathrm{PrOH}, 1.0$ $\mathrm{mL} / \mathrm{min}, \lambda=254 \mathrm{~nm}$, $97 \%$ ee.

\section{Authentic racemic}

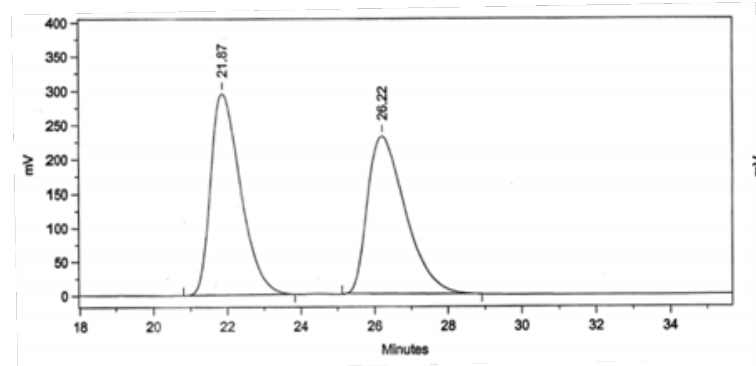

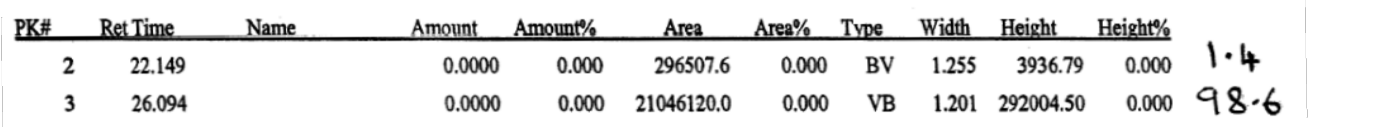

Characterization data for pyran products: Oxabicyles $8,10,12,14$, and 16 were prepared according to published literature procedures and characterization data for pyrans $9,11,13,15$, and 17 was previously reported. ${ }^{7}$

(7) For HPLC traces of reactions in Table 2 with Ru complex $\mathbf{1 b}$, see supporting information in: Gillingham, D. G.; Kataoka, O.; Garber, S. B.; Hoveyda, A. H. J. Am. Chem. Soc. 2004, 126, 12288-12290. 
Pyran 9. The enantiomeric purity of this compound was determined by HPLC analysis in comparison with authentic racemic material, shown below: Chiralpak OB-H (4.6 x 250 $\mathrm{mm})$, 99.8:0.2 hexanes: $i-\mathrm{PrOH}, 0.5 \mathrm{~mL} / \mathrm{min}, \lambda=254 \mathrm{~nm}$.

\section{Authentic racemic}

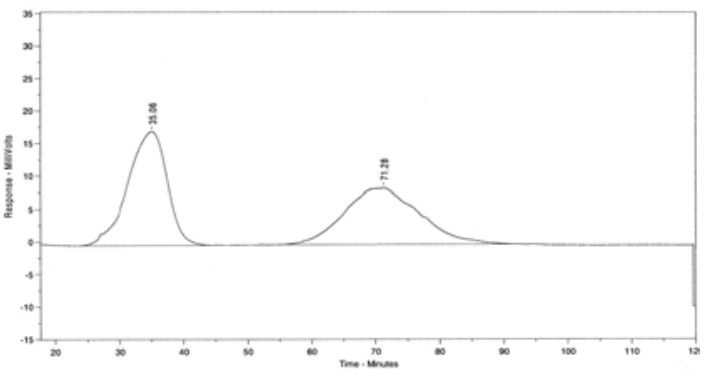

Authentic racemic

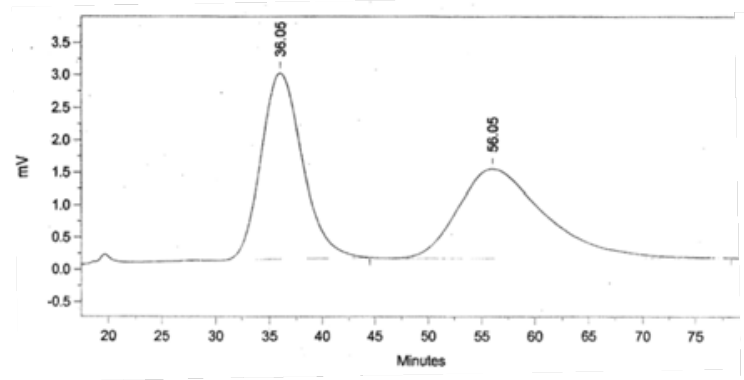

\begin{tabular}{rrrrrrrrrrr} 
PK\# & Ret Time & Name & Amount & Amount\% & \multicolumn{1}{c}{ Area } & Area\% & Type & Width & Height & Height\% \\
1 & 35.967 & 0.0000 & 0.000 & 266301.7 & 1.507 & BB & 3.333 & 1331.49 & 3.932 \\
2 & 56.105 & 0.0000 & 0.000 & 17399460.0 & 98.493 & BB & 8.914 & 32531.87 & 96.068
\end{tabular}

\section{$>98 \%$ ee with $\mathrm{Ru}$ catalyst $\mathbf{2 b}$}

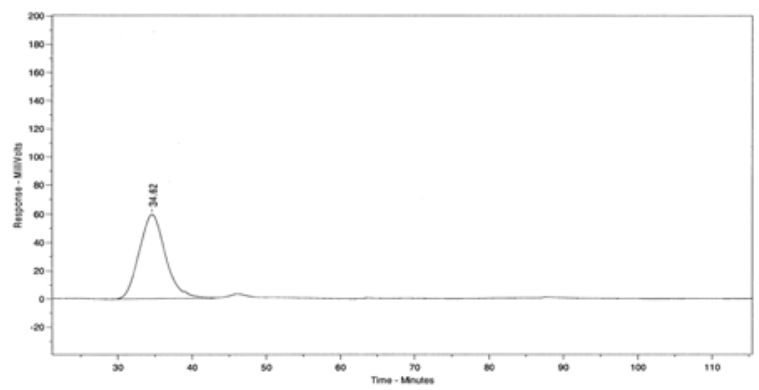

97\% ee with Mo catalyst 6

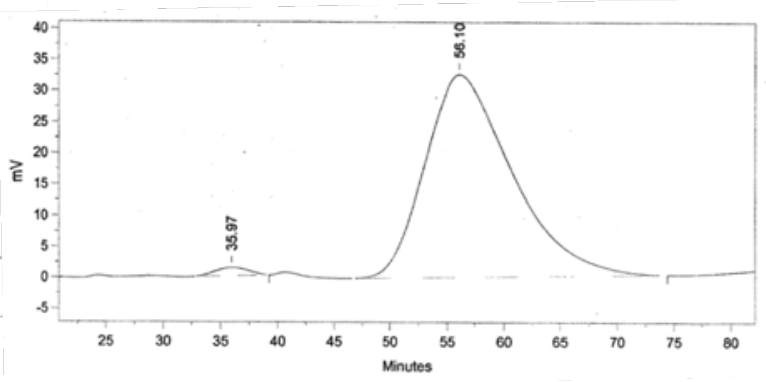


Pyran 11. The enantiomeric purity of this compound was determined by HPLC analysis in comparison with authentic racemic material, shown below: Chiralpak OJ (4.6 x 250 $\mathrm{mm}), 99: 1$ hexanes: $i-\mathrm{PrOH}, 1.0 \mathrm{~mL} / \mathrm{min}, \lambda=254 \mathrm{~nm}$.

Authentic racemic

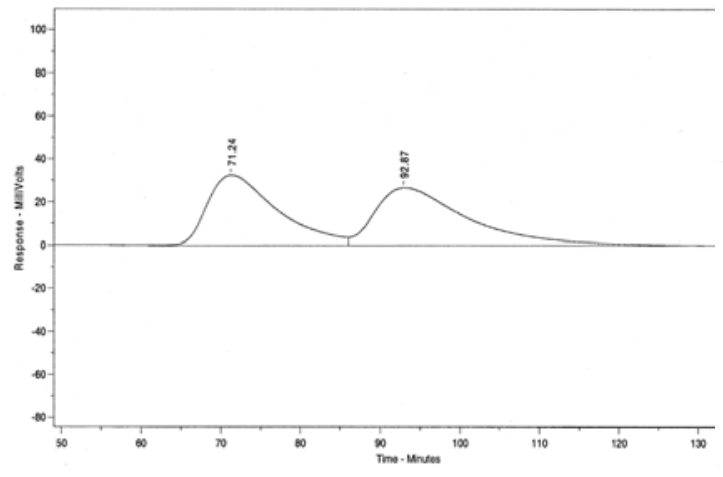

Peak \# Ret. Time Name

$\begin{array}{lr}1 & 72.31 \\ 2 & 95.95\end{array}$
$88 \%$ ee with $\mathrm{Ru}$ catalyst $2 \mathrm{~b}$ at $22{ }^{\circ} \mathrm{C}$

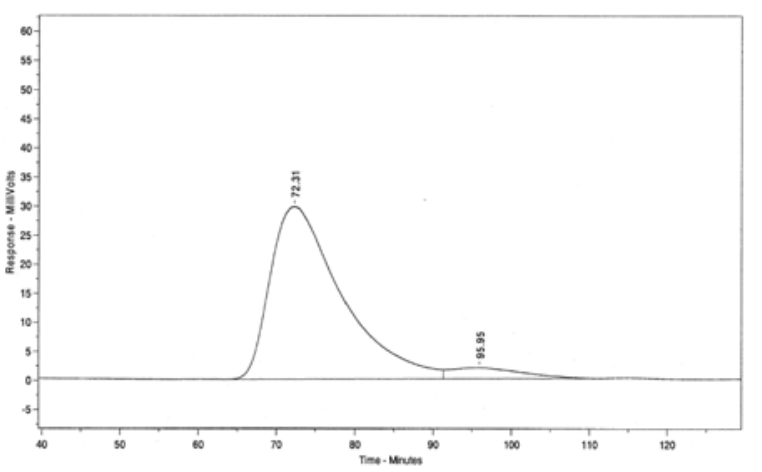

Authentic racemic

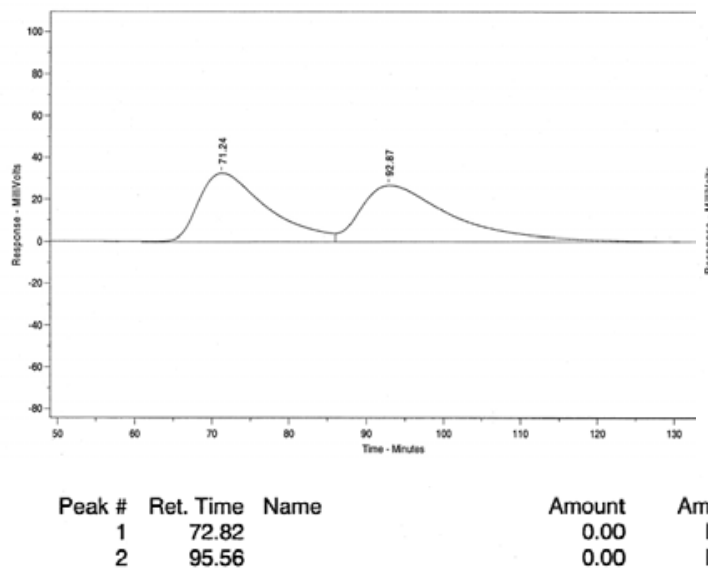

$91 \%$ ee with $\mathrm{Ru}$ catalyst $2 \mathrm{~b}$ at $-15{ }^{\circ} \mathrm{C}$

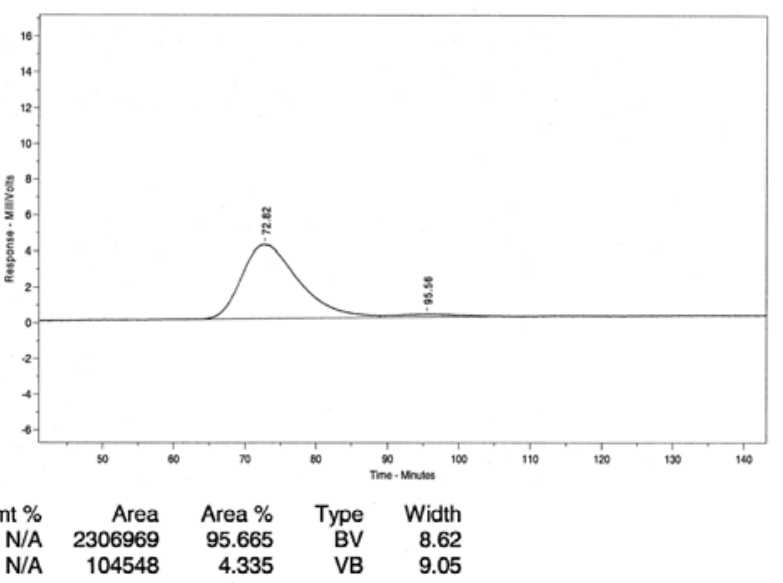

Pyran 13. The enantiomeric purity of this compound was determined by HPLC analysis in comparison with authentic racemic material, shown below: Chiralpak OJ (4.6 x 250 $\mathrm{mm})$, 99.5:0.5 hexanes: $i-\mathrm{PrOH}, 1.0 \mathrm{~mL} / \mathrm{min}, \lambda=254 \mathrm{~nm}$.

\section{Authentic racemic}



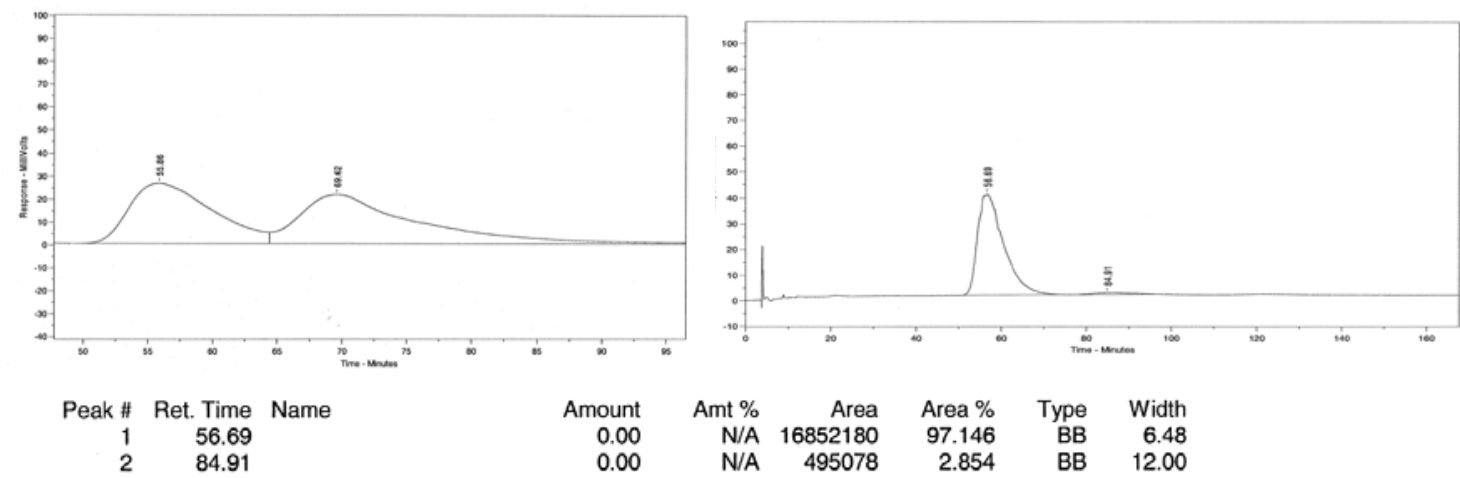

\section{Authentic racemic}

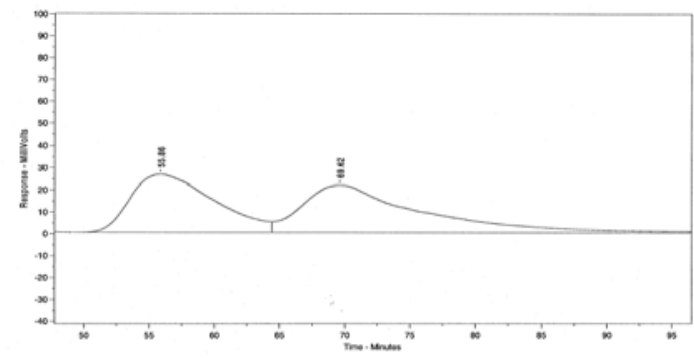

$>98 \%$ ee with $\mathrm{Ru}$ catalyst $2 \mathrm{~b}$ at $-15^{\circ} \mathrm{C}$

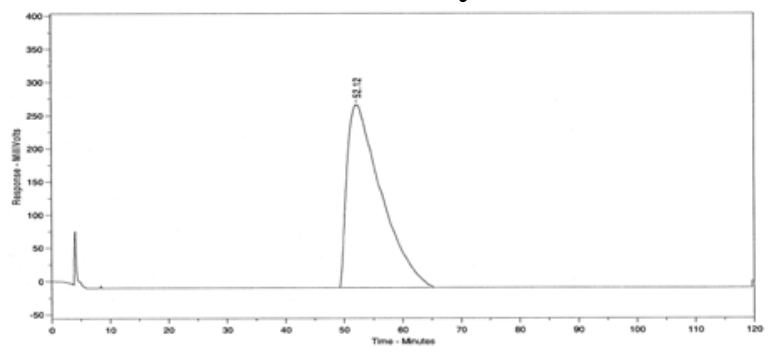

Authentic racemic
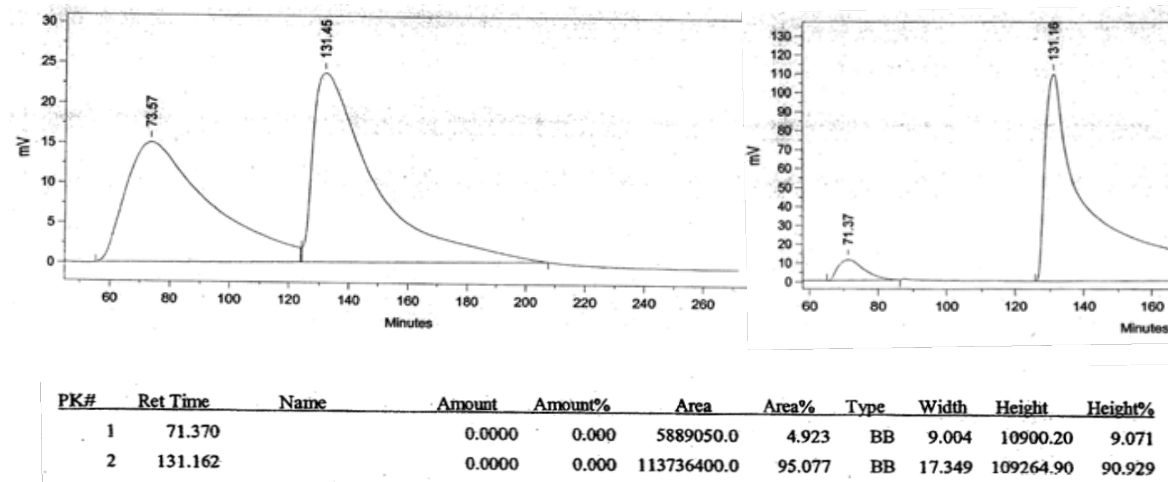

90\% ee with Mo catalyst 6

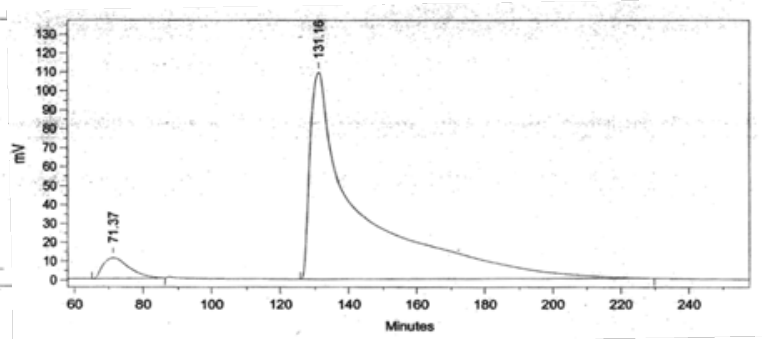

$\begin{array}{lllll}95.077 & \text { BB } & 17.349 & 109264.90 & 90.929\end{array}$

Pyran 15. The enantiomeric purity of this compound was determined by HPLC analysis in comparison with authentic racemic material, shown below: Chiralpak OJ (4.6 x 250 $\mathrm{mm})$, 99.8:0.2 hexanes: $i-\mathrm{PrOH}, 1.0 \mathrm{~mL} / \mathrm{min}, \lambda=254 \mathrm{~nm}$.

\section{Authentic racemic}

$82 \%$ ee with $\mathrm{Ru}$ catalyst $2 \mathrm{~b}$ 


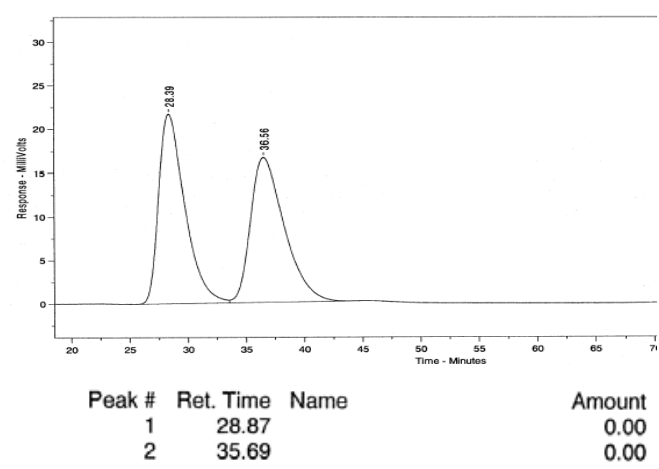

Authentic racemic
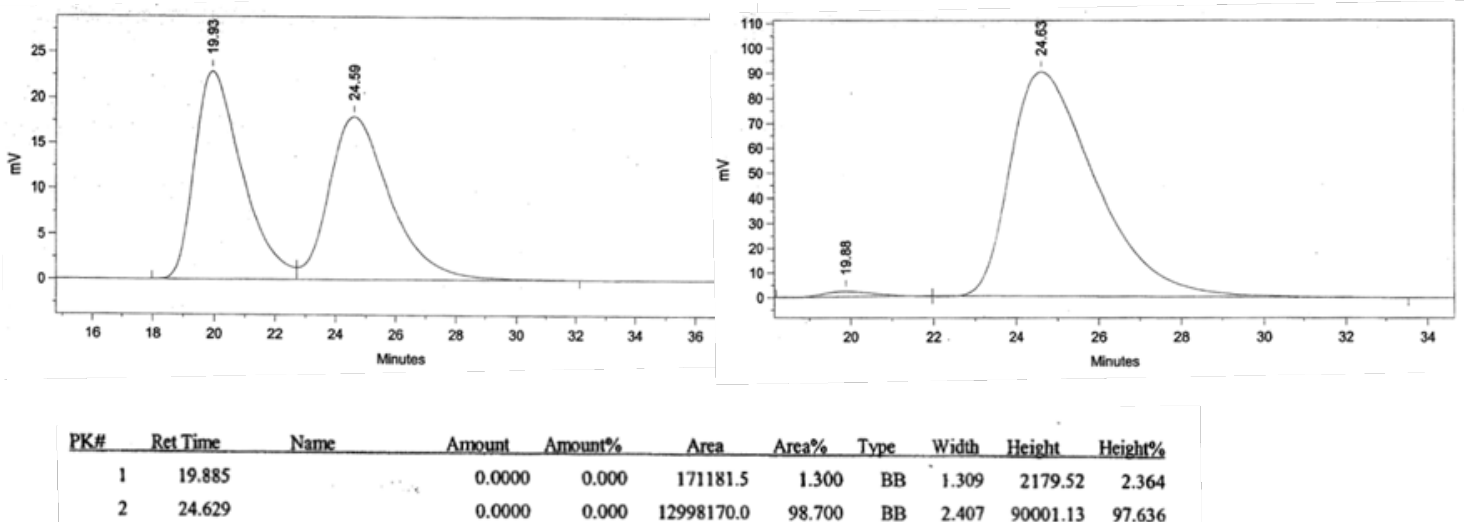

Pyran 17. The enantiomeric purity of this compound was determined by HPLC analysis in comparison with authentic racemic material, shown below: Chiralpak AD (4.6 x 250 $\mathrm{mm}), 96: 4$ hexanes: $i-\mathrm{PrOH}, 0.8 \mathrm{~mL} / \mathrm{min}, \lambda=210 \mathrm{~nm}, 67 \%$ ee.

\section{Authentic racemic}
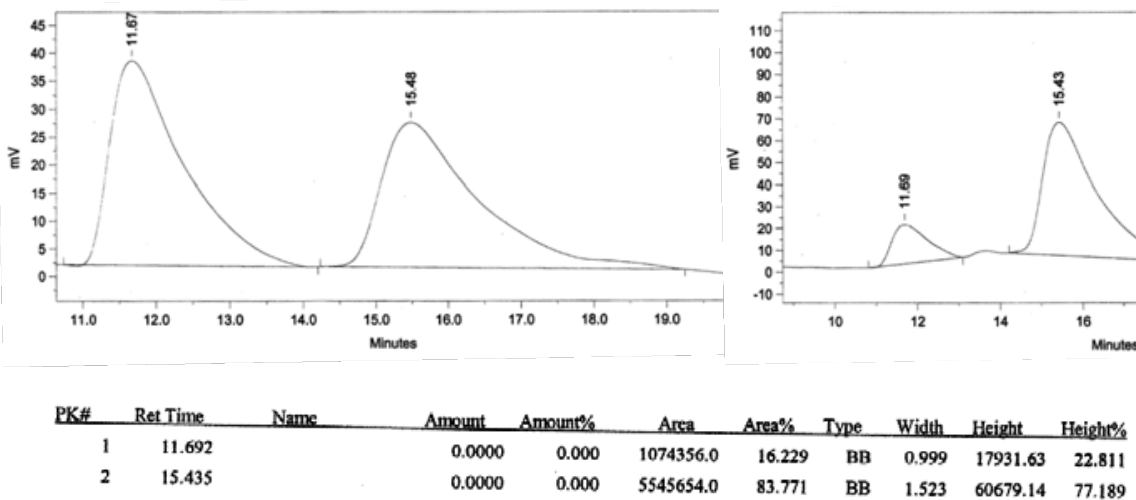

\section{$67 \%$ ee with Mo catalyst 6}

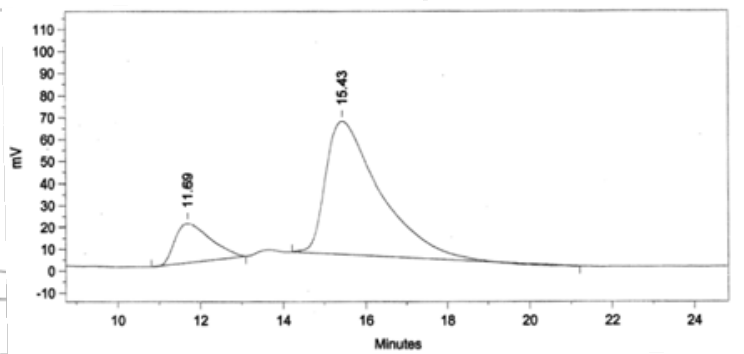

\section{Characterization data for piperidine products:}

Piperidine 23b. Method A for Ru-catalyzed AROM/CM was followed. ${ }^{1} \mathrm{H}$ NMR (400 $\left.\mathrm{MHz}, \mathrm{CDCl}_{3}\right): \delta 7.49(\mathrm{~d}, J=7.6 \mathrm{~Hz}, 1 \mathrm{H}), 7.39-7.25(\mathrm{~m}, 4 \mathrm{H}), 7.11(\mathrm{dd}, J=7.6 \mathrm{~Hz}, 1 \mathrm{H})$, $7.03(\mathrm{dd}, J=7.6 \mathrm{~Hz}, 1 \mathrm{H}), 6.84(\mathrm{dd}, J=8.0 \mathrm{~Hz}, 1 \mathrm{H}), 6.78(\mathrm{~d}, J=16.0 \mathrm{~Hz}, 1 \mathrm{H}), 5.90-5.79$ (m, 2H), 5.20 (d, $J=17.2 \mathrm{~Hz}, 1 \mathrm{H}), 5.08$ (dd, $J=10.0,1.6 \mathrm{~Hz}, 1 \mathrm{H}), 3.99$ (d, $J=15.6 \mathrm{~Hz}$, 
$1 \mathrm{H}), 3.80-3.70(\mathrm{~m}, 1 \mathrm{H}), 3.68(\mathrm{~d}, J=15.6 \mathrm{~Hz}, 1 \mathrm{H}), 3.18-3.03(\mathrm{~m}, 2 \mathrm{H}), 1.96-1.92(\mathrm{~m}$, 2H), 1.56-1.48 (m, 3H). $\left.).{ }^{13} \mathrm{C} \mathrm{NMR} \mathrm{(100} \mathrm{MHz,} \mathrm{CDCl}_{3}\right): \delta 142.4,140.1,137.3,137.0$, $132.8,129.4,129.2,128.9,128.7,128.2,128.0,127.4,127.3,126.3,123.4,116.2,67.9$, 64.3, 64.2, 55.4, 43.1, 42.9, 42.8. HRMS ES $(\mathrm{m} / \mathrm{z})$ mass Calcd for $\mathrm{C}_{22} \mathrm{H}_{25} \mathrm{BrNO} 398.1120$ $(\mathrm{M}+\mathrm{H})^{+}$, Found 398.1113. The enantiomeric purity of this compound was determined by HPLC analysis in comparison with authentic racemic material, shown below: Chiralpak OD (4.6 x $250 \mathrm{~mm}), 98: 2$ hexanes: $i-\mathrm{PrOH}, 1.0 \mathrm{~mL} / \mathrm{min}, \lambda=254 \mathrm{~nm}$, $97 \%$ ee.

\section{Authentic racemic}
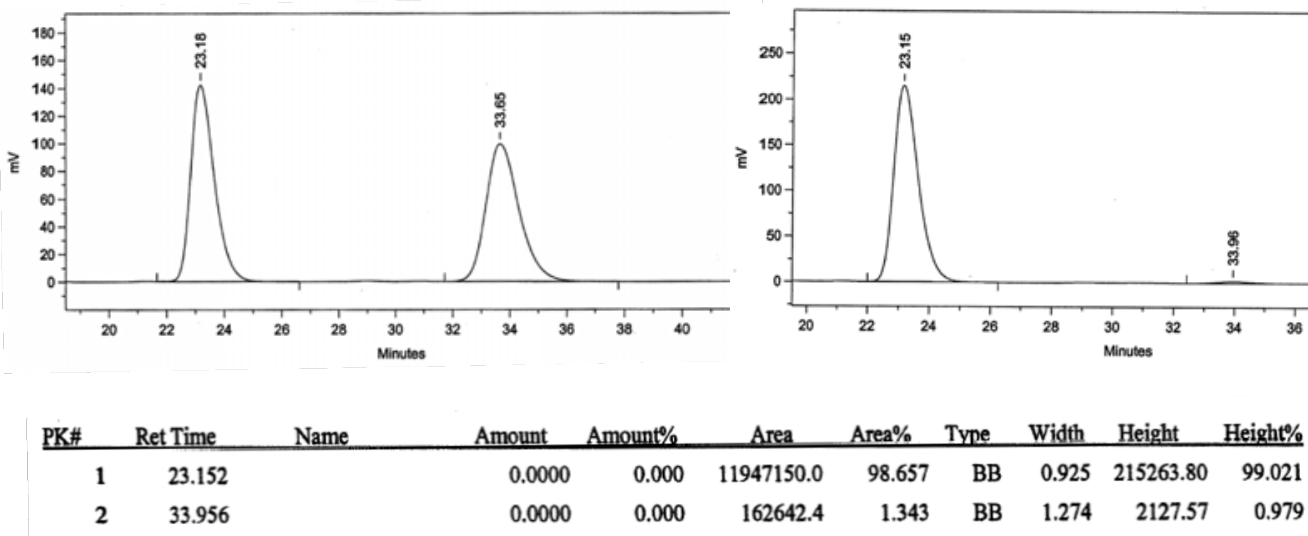

Piperidine 23c. Method A for Ru-catalyzed AROM/CM was followed. IR (neat): 3333 (br), 2930 (w), 2917 (w), 2836 (w), 1483 (w), 1451 (m), 1086 (s). ${ }^{1}$ H NMR (400 MHz, $\left.\mathrm{CDCl}_{3}, \mathrm{TMS}\right): \delta 7.32(\mathrm{~d}, J=7.0 \mathrm{~Hz}, 2 \mathrm{H}), 7.27-7.17(\mathrm{~m}, 3 \mathrm{H}), 7.10-7.03(\mathrm{~m}, 3 \mathrm{H}), 6.92(\mathrm{~d}$, $J=7.5 \mathrm{~Hz}, 1 \mathrm{H}), 6.66(\mathrm{~d}, J=15.8 \mathrm{~Hz}, 1 \mathrm{H}), 5.90(\mathrm{dd}, J=15.8,8.6 \mathrm{~Hz}, 1 \mathrm{H}), 5.83$ (ddd, $J=$ 17.4, 10.1, 8.4 Hz, 1H), 5.21 (dd, $J=17.4,0.9 \mathrm{~Hz}, 1 \mathrm{H}), 5.07$ (dd, $J=10.1,1.3 \mathrm{~Hz}, 1 \mathrm{H})$, $3.97(\mathrm{~d}, J=15.4 \mathrm{~Hz}, 1 \mathrm{H}), 3.78$ (d, $J=15.4 \mathrm{~Hz}, 1 \mathrm{H}), 3.77-3.70(\mathrm{~m}, 1 \mathrm{H}), 3.13$ (ddd, $J=$ 11.2, 8.6, $2.3 \mathrm{~Hz}, 1 \mathrm{H}), 3.06$ (ddd, $J=11.2,8.4,2.6 \mathrm{~Hz}, 1 \mathrm{H}), 2.31$ (s, 3H), 1.97-1.91 (m, $2 \mathrm{H}), 1.64$ (br s, $1 \mathrm{H}), 1.60-1.48(\mathrm{~m}, 2 \mathrm{H}) .{ }^{13} \mathrm{C} \mathrm{NMR}\left(100 \mathrm{MHz}, \mathrm{CDCl}_{3}\right): \delta 142.4,140.0$, 136.2, 135.6, 135.1, 130.1, 129.1, 128.1, 127.9, 127.3, 126.3, 126.1, 125.9, 115.9, 68.0, 64.3, 64.2, 55.1, 43.2, 43.0, 19.8. HRMS ES $(\mathrm{m} / \mathrm{z})$ mass Calcd for $\mathrm{C}_{23} \mathrm{H}_{29} \mathrm{NO} 334.2171$ $(\mathrm{M}+\mathrm{H})^{+}$, Found 334.2176. $[\alpha]_{\mathrm{D}}{ }^{25}-106.2\left(c 1.0, \mathrm{CHCl}_{3}\right)$ for a sample of $>98 \%$ ee. The enantiomeric purity of this compound was determined by HPLC analysis in comparison with authentic racemic material, shown below: Chiralpak OD (4.6 x $250 \mathrm{~mm}), 98: 2$ hexanes: $i$-PrOH, $1.0 \mathrm{~mL} / \mathrm{min}, \lambda=254 \mathrm{~nm},>98 \%$ ee. 

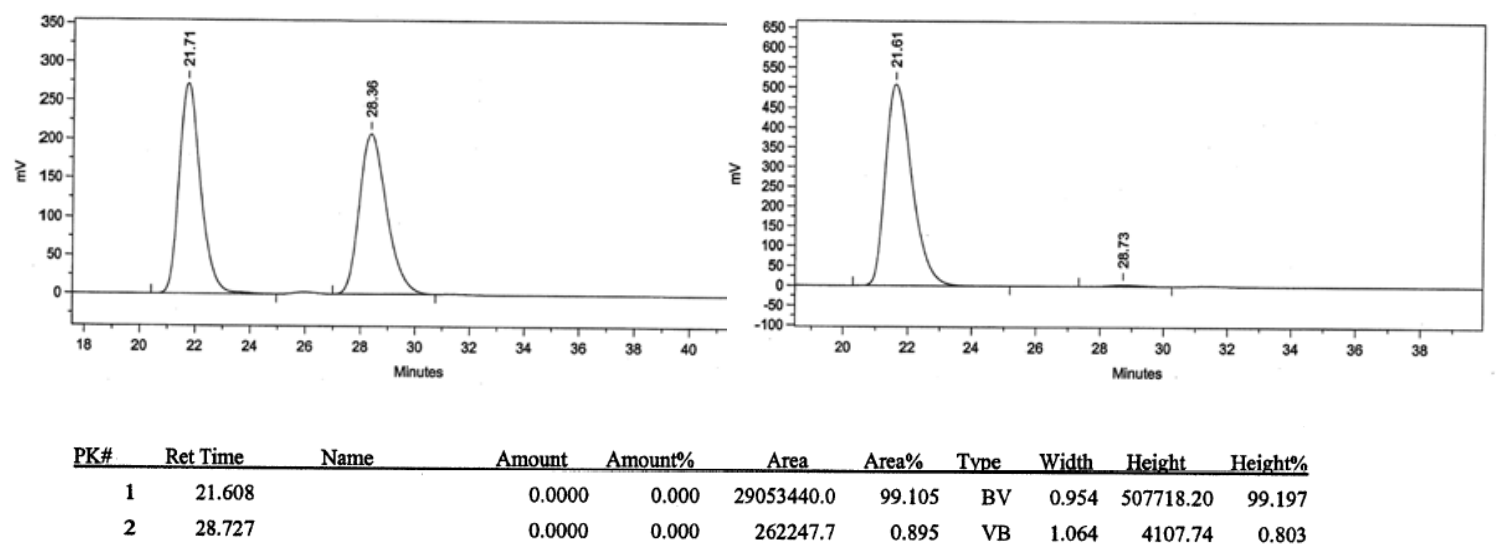

\section{Authentic racemic}

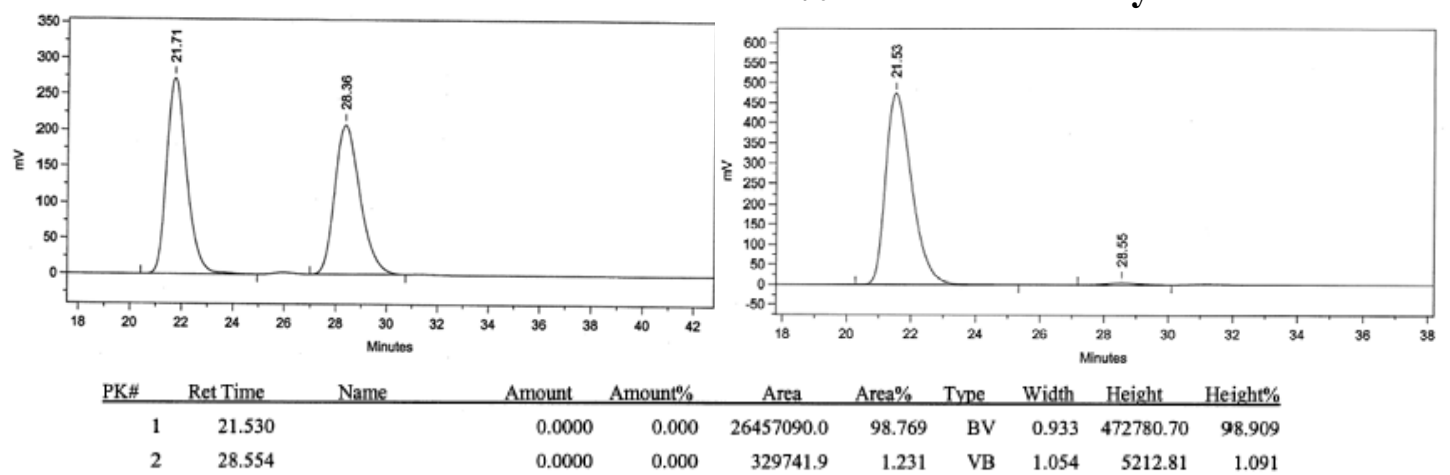

Piperidine 23d. Method A for Ru-catalyzed AROM/CM was followed. ${ }^{1} \mathrm{H}$ NMR (400 $\left.\mathrm{MHz}, \mathrm{CDCl}_{3}\right): \delta 7.31-7.12(\mathrm{~m}, 6 \mathrm{H}), 7.00-6.96(\mathrm{~m}, 3 \mathrm{H}) 6.62(\mathrm{~d}, J=16.0 \mathrm{~Hz}, 1 \mathrm{H}), 6.00$ (dd, $J=16.0,8.8 \mathrm{~Hz}, 1 \mathrm{H}), 5.82(\mathrm{ddd}, J=17.2,10.0,8.4 \mathrm{~Hz}, 1 \mathrm{H}), 5.20$ (dd, $J=17.2,0.8$ $\mathrm{Hz}, 1 \mathrm{H}), 5.06(\mathrm{dd}, J=10.4,1.6 \mathrm{~Hz}, 1 \mathrm{H}), 3.95(\mathrm{~d}, J=15.6 \mathrm{~Hz}, 1 \mathrm{H}), 3.75-3.72(\mathrm{~m}, 1 \mathrm{H})$, 3.69 (d, $J=15.2 \mathrm{~Hz}, 1 \mathrm{H}), 3.11$ (ddd, $J=11.2,8.8,2.4 \mathrm{~Hz}, 1 \mathrm{H}), 3.05$ (ddd, $J=11.2,8.8$, $2.4 \mathrm{~Hz}, 1 \mathrm{H}), 1.95-1.90(\mathrm{~m}, 2 \mathrm{H}), 1.58-1.46(\mathrm{~m}, 3 \mathrm{H}) .{ }^{13} \mathrm{C} \mathrm{NMR}\left(100 \mathrm{MHz}, \mathrm{CDCl}_{3}\right): \delta$ $161.3,158.8,142.4,140.2$, 136.6, 129.4, 129.2, 128.7, 128.6, 128.2, 128.0, 127.2, 127.1, $126.3,124.9,124.8,124.1,122.4,116.1,115.6,115.4,68.0,64.5,64.4,55.4,43.0,42.9$. HRMS ES $(\mathrm{m} / \mathrm{z})$ mass Calcd for $\mathrm{C}_{22} \mathrm{H}_{25}$ NOF $338.1920(\mathrm{M}+\mathrm{H})^{+}$, Found 338.1906. 

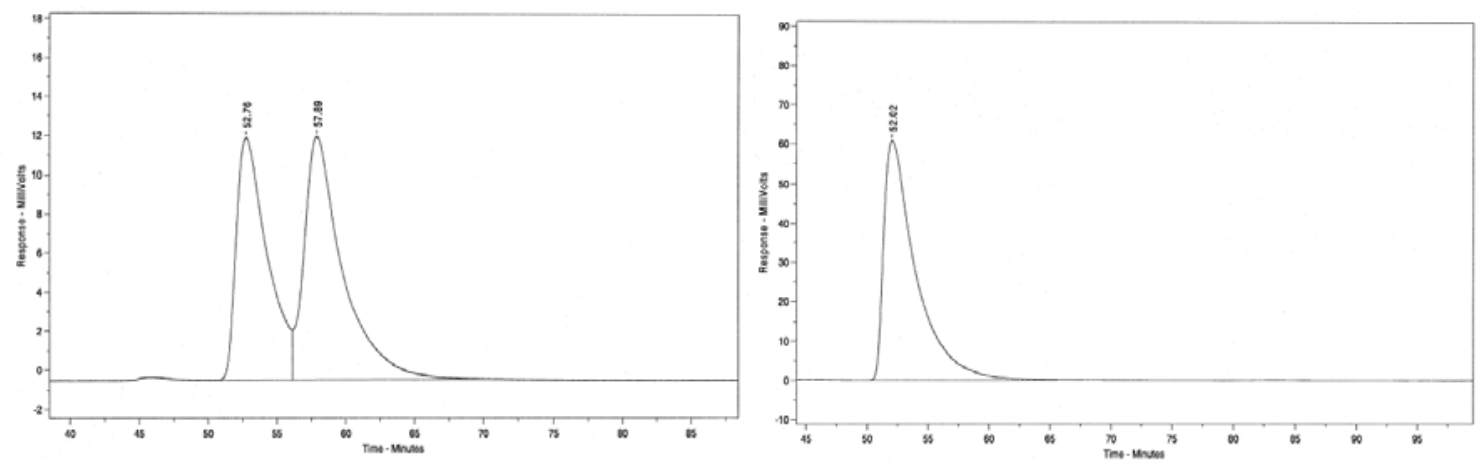

Piperidine 23e. Method A for Ru-catalyzed AROM/CM was followed. IR (neat): 3355 (br), 2920 (m), 2839 (m), 1604 (m), 1511 (s), 1251 (m). ${ }^{1} \mathrm{H}$ NMR (400 MHz, CDCl TMS): $\delta 7.28-7.14(\mathrm{~m}, 5 \mathrm{H}), 7.05(\mathrm{~d}, J=8.8 \mathrm{~Hz}, 2 \mathrm{H}), 6.78(\mathrm{~d}, J=8.8 \mathrm{~Hz}, 2 \mathrm{H}), 6.38(\mathrm{~d}, J$ $=15.9 \mathrm{~Hz}, 1 \mathrm{H}), 5.83(\mathrm{dd}, J=15.9,8.6 \mathrm{~Hz}, 1 \mathrm{H}), 5.81(\mathrm{ddd}, J=17.4,10.3,8.4 \mathrm{~Hz}, 1 \mathrm{H})$, $5.19(\mathrm{dd}, J=17.4,0.9 \mathrm{~Hz}, 1 \mathrm{H}), 5.05(\mathrm{dd}, J=10.3,1.5 \mathrm{~Hz}, 1 \mathrm{H}), 3.93(\mathrm{~d}, J=15.4 \mathrm{~Hz}, 1 \mathrm{H})$, $3.78(\mathrm{~s}, 3 \mathrm{H}), 3.75-3.67(\mathrm{~m}, 1 \mathrm{H}), 3.73(\mathrm{~d}, J=15.4 \mathrm{~Hz}, 1 \mathrm{H}), 3.07$ (ddd, $J=11.2,8.6,2.4$ $\mathrm{Hz}, 1 \mathrm{H}), 3.03$ (ddd, $J=11.2,8.4,2.6 \mathrm{~Hz}, 1 \mathrm{H}), 1.94-1.90(\mathrm{~m}, 2 \mathrm{H}), 1.62$ (br s, $1 \mathrm{H})$, 1.57-1.46 (m, 2H). $\left.{ }^{13} \mathrm{C} \mathrm{NMR} \mathrm{(100} \mathrm{MHz,} \mathrm{CDCl}_{3}\right): \delta 159.1,142.5,140.3,132.1,130.0$, $129.7,129.3,127.9,127.6,126.3,115.9,114.0,68.1,64.5,64.2,55.4,55.2,43.2,43.2$. HRMS ES $(m / z)$ mass Calcd for $\mathrm{C}_{23} \mathrm{H}_{28} \mathrm{NO}_{2} 350.2120(\mathrm{M}+\mathrm{H})^{+}$, Found 350.2118. $[\alpha]_{\mathrm{D}}{ }^{25}$ $-158.0\left(c 1.0, \mathrm{CHCl}_{3}\right)$ for a sample of $98 \%$ ee. The enantiomeric purity of this compound was determined by HPLC analysis in comparison with authentic racemic material, shown below: Chiralpak OD (4.6 x $250 \mathrm{~mm}$ ), 98:2 hexanes: $i$-PrOH, $1.0 \mathrm{~mL} / \mathrm{min}, \lambda=254 \mathrm{~nm}$, 98\% ee with Ru complex 1a and 96\% ee with Ru complex $\mathbf{2 a}$.

\section{Authentic racemic}

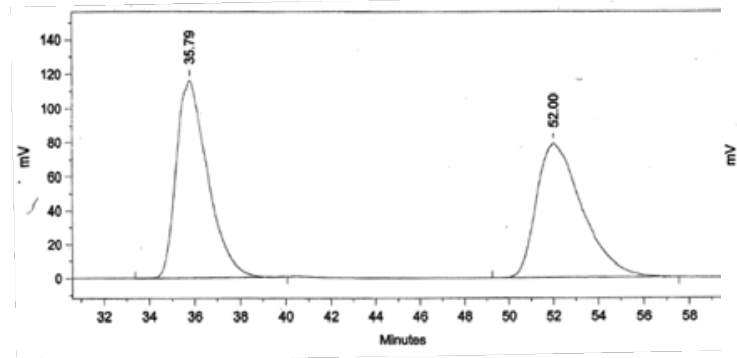

98\% ee with Ru catalyst 1a

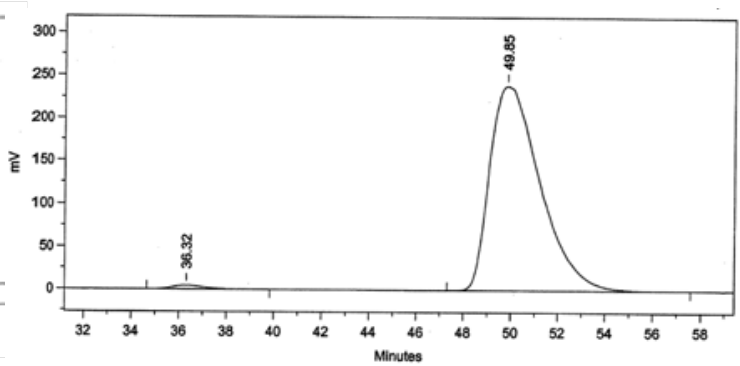

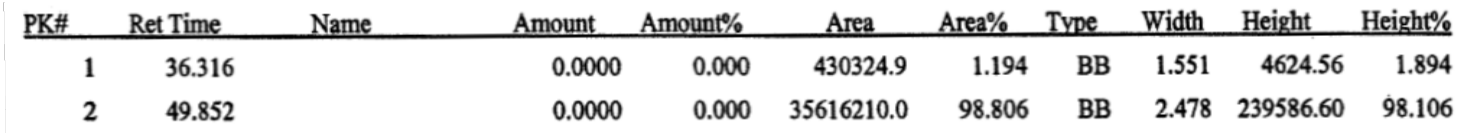


Authentic racemic

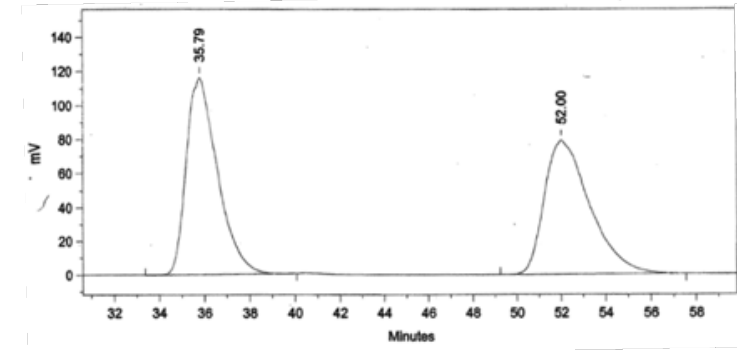

$96 \%$ ee with Ru catalyst 2a

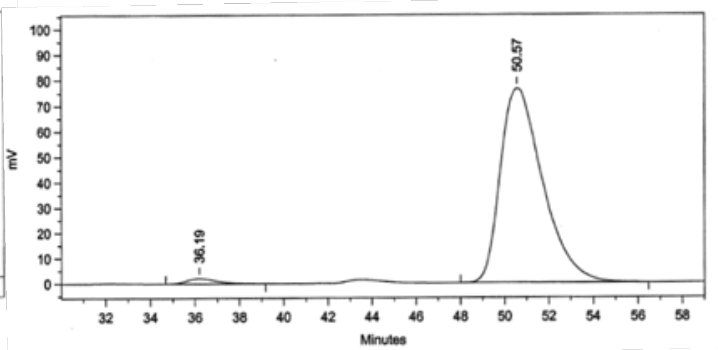

$\underline{\text { PK }}$

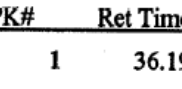

Name

$\begin{array}{rr}\text { Amount } & \text { Amount\% } \\ 0.0000 & 0.000 \\ 0.0000 & 0.000\end{array}$

\begin{tabular}{rrrrrr} 
Area & Area\% & Type & Width & Height & Height\% \\
\hline 191776.8 & 1.838 & BB & 1.542 & 2072.18 & 2.633 \\
10243310.0 & 98.162 & BB & 2.228 & 76631.08 & 97.367
\end{tabular}

\section{Scheme 1. Synthesis of Azabicycle 22}<smiles>COc1ccncc1</smiles>

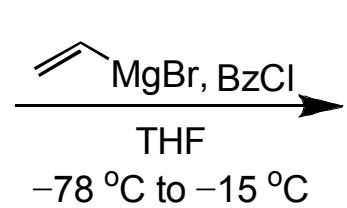
$1 \mathrm{~h}$<smiles>C=C[C@@H]1CC(=O)C=CN1C(=O)c1ccccc1</smiles>

A $96 \%$ yield

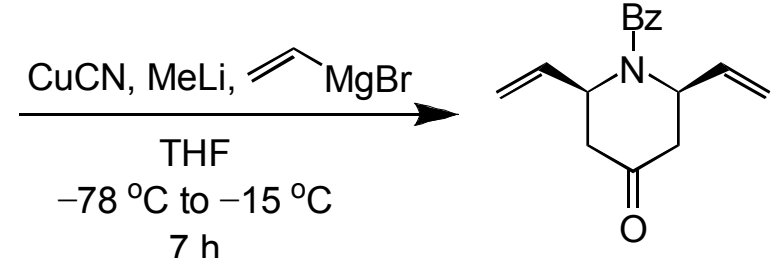

B $90 \%$ yield<smiles></smiles>

$18 \mathrm{~h}$

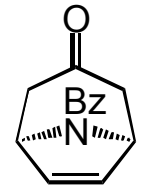

C $60 \%$ yield
1) L-Selectride, THF, $-78^{\circ} \mathrm{C}$ to $22^{\circ} \mathrm{C}, 1 \mathrm{~h}$

2) DIBAI-H, THF, $-78{ }^{\circ} \mathrm{C}$ to $22{ }^{\circ} \mathrm{C}, 3 \mathrm{~h}$<smiles>O[C@H]1C[C@@H]2C=C[C@@H]1C[C@@H]2Br</smiles>

22

$40 \%$ yield over two steps

- Synthesis of Azabicycles: The synthesis of azabicyclic substrate 18 has been previously reported. $^{8}$ A modified synthetic route to $\mathbf{1 8}$ was employed for the preparation of azabicycle 22 and is shown in Scheme 1. Alkylation of $p$-methoxypyridine with vinylmagnesium bromide in the presence of benzoyl chloride provided piperidinone $\mathbf{A}$. Conjugate addition to $\mathbf{A}$ with vinyl cuprate reagent then provided divinyl piperidinone $\mathbf{B}$. Ring-closing metathesis of $\mathbf{B}$ with $\mathrm{Ru}$ catalyst $\mathbf{D}^{9}$ furnished azabicycle $\mathbf{C}$. Selective

(8) Cortez, G. A.; Schrock, R. R.; Hoveyda, A. H. Angew. Chem., Int. Ed. 2007, in press.

(9) Garber, S. B.; Kingsbury, J. S.; Gray, B. L.; Hoveyda, A. H. J. Am. Chem. Soc. 2000, 122, 8168-8179. 
reduction of the carbonyl in $\mathbf{C}$ with L-Selectride ${ }^{\circledR}$ followed by reduction of the benzamide to the tertiary benzyl amine provided metathesis substrate $\mathbf{2 2}$.

Synthesis of Piperidinone A. A 250-mL round-bottom flask, fitted with an addition funnel, was charged with $p$-methoxypyridine $(5.00 \mathrm{~g}, 46.2 \mathrm{mmol})$, THF (46 mL), and the reaction vessel was allowed to cool to $-78{ }^{\circ} \mathrm{C}$ in a dry ice/acetone bath. To this solution was added vinylmagnesium bromide $(55.5 \mathrm{~mL}, 55.5 \mathrm{mmol}, 1.0 \mathrm{M}$ in THF) dropwise over a period of $10 \mathrm{~min}$, after which time the resulting mixture was allowed to warm to $-15{ }^{\circ} \mathrm{C}$ in a cooling bath. At this time, to this mixture was added benzoyl chloride $(8.48 \mathrm{~mL}$, $69.4 \mathrm{mmol}$ ) dropwise over a period of $10 \mathrm{~min}$. The mixture was allowed to stir at $-15^{\circ} \mathrm{C}$ for $20 \mathrm{~min}$, after which time the flask was removed from the cooling bath and the reaction was quenched by the addition of a $2.0 \mathrm{M}$ solution of $\mathrm{HCl}(75 \mathrm{~mL})$. The resulting solution was allowed to stir at $22{ }^{\circ} \mathrm{C}$ for $15 \mathrm{~min}$, after which time it was washed with $\mathrm{Et}_{2}$ $\mathrm{O}(4 \times 100 \mathrm{~mL})$. The combined organic layers were washed with a $1.0 \mathrm{M}$ solution of $\mathrm{NaOH}(25 \mathrm{~mL})$, a saturated aqueous solution of $\mathrm{NaCl}(50 \mathrm{~mL})$, dried $\left(\mathrm{MgSO}_{4}\right)$, filtered, and the volatiles removed in vacuo. The dark brown residue was purified by silica gel chromatography (dry load, 1:1 to $3: 1 \mathrm{Et}_{2} \mathrm{O}$ :hexanes) to give $\mathbf{A}$ as slight yellow solid (10.05 g, $44.20 \mathrm{mmol}, 96 \%)$. mp: 40-43 ${ }^{\circ} \mathrm{C}$. IR (neat): 3081 (w), 2995 (w), 1666 (s), 1598 (s), 1393 (m), 1331 (s), 1288 (s), 1213 (s), 1145 (s). ${ }^{1} \mathrm{H}$ NMR (400 MHz, CDCl TMS): $\delta 7.56-7.45(\mathrm{~m}, 6 \mathrm{H}), 5.87(\mathrm{ddd}, J=17.2,10.6,4.9 \mathrm{~Hz}, 1 \mathrm{H}), 5.44-5.40(\mathrm{~m}, 1 \mathrm{H})$, 5.30-5.27 (m, 2H), $5.21(\mathrm{dd}, J=17.2,1.6 \mathrm{~Hz}, 1 \mathrm{H}), 2.98(\mathrm{dd}, J=16.7,6.6 \mathrm{~Hz}, 1 \mathrm{H}), 2.68$ $(\mathrm{d}, J=16.7 \mathrm{~Hz}, 1 \mathrm{H}) .{ }^{13} \mathrm{C} \mathrm{NMR}\left(100 \mathrm{MHz}, \mathrm{CDCl}_{3}\right): \delta 192.6,172.0,142.8,132.9,132.7$, $131.8,128.9,128.5,118.0,107.8,54.4,40.2$. HRMS ES $(m / z)$ Calcd for $\mathrm{C}_{14} \mathrm{H}_{14} \mathrm{NO}_{2}$ $228.1025(\mathrm{M}+\mathrm{H})^{+}$, Found 228.1024.

Synthesis of cis-2-Vinylpiperidine B. A 500-mL round bottom flask, fitted with an addition funnel, was charged with $\mathrm{CuCN}$ (2.96 g, $33.1 \mathrm{mmol})$, THF (51 mL), and the reaction vessel was allowed to cool to $-78{ }^{\circ} \mathrm{C}$ in a dry ice/acetone bath. To this solution was added MeLi (21.4 mL, $\left.33.1 \mathrm{mmol}, 1.54 \mathrm{M}^{\text {in }} \mathrm{Et}_{2} \mathrm{O}\right)$ dropwise over a period of 10 min. At this time, the reaction flask was removed from the dry ice bath and placed in an ice bath at $0{ }^{\circ} \mathrm{C}$ for $1 \mathrm{~min}$, after which time the flask was allowed to cool to $-78{ }^{\circ} \mathrm{C}$ in a dry ice/acetone bath. To this mixture was added vinylmagnesium bromide $(34.0 \mathrm{~mL}$, $33.1 \mathrm{mmol}, 0.97 \mathrm{M}$ in THF, dropwise over a period of $15 \mathrm{~min}$ and the mixture was allowed to stir at $-78{ }^{\circ} \mathrm{C}$ for $10 \mathrm{~min}$. The mixture was then charged with a solution of $\mathbf{A}$ $(5.00 \mathrm{~g}, 22.0 \mathrm{mmol}$, as a solution in $20 \mathrm{~mL}$ of THF) in a dropwise fashion over a period of $40 \mathrm{~min}$, and the resulting orange mixture was allowed to stir at $-78{ }^{\circ} \mathrm{C}$ for $5 \mathrm{~h}$ (it is critical to add A slowly to prevent formation of the trans product). At this time, the mixture was poured into a solution consisting of a saturated aqueous solution of $\mathrm{NH}_{4} \mathrm{Cl}$ and a saturated aqueous solution of $\mathrm{NH}_{4} \mathrm{OH}(9: 1,250 \mathrm{~mL})^{10}$ at $22{ }^{\circ} \mathrm{C}$. The mixture was

(10) Caution should be taken as this part of the procedure is exothermic. 
allowed to stir for $12 \mathrm{~h}$, after which time the solution was washed with EtOAc $(3 \times 250$ $\mathrm{mL})$. The combined organic layers were washed with a saturated aqueous solution of $\mathrm{NaCl}(150 \mathrm{~mL})$, dried $\left(\mathrm{MgSO}_{4}\right)$, filtered, and the volatiles removed in vacuo. The resulting dark brown residue was purified by silica gel chromatography (dry load, 4:1 to 1:1 hexanes:EtOAc) to provide $\mathbf{B}$ as colorless oil (5.05 g, $19.8 \mathrm{mmol}, 90 \%)$. IR (neat): 3081 (w), 2974 (w), 2905 (w), 1722 (s), 1634 (s), 1394 (s), 1338 (s), 1224 (m), 916 (m), $702(\mathrm{~m}) .{ }^{1} \mathrm{H}$ NMR $\left(400 \mathrm{MHz}, \mathrm{CDCl}_{3}, \mathrm{TMS}\right): \delta 7.48-7.41(\mathrm{~m}, 5 \mathrm{H}), 5.98(\mathrm{ddd}, J=17.2$, 10.6, $5.1 \mathrm{~Hz}, 2 \mathrm{H}), 5.23-5.18(\mathrm{~m}, 6 \mathrm{H}), 2.69(\mathrm{~d}, J=5.7 \mathrm{~Hz}, 4 \mathrm{H}) .{ }^{13} \mathrm{C} \mathrm{NMR}(100 \mathrm{MHz}$, $\left.\mathrm{CDCl}_{3}\right): \delta 206.1,172.0,138.2,136.1,130.2,128.9,128.7,126.6,117.2,54.7,43.2$. HRMS ES $(m / z)$ mass Calcd for $\mathrm{C}_{16} \mathrm{H}_{18} \mathrm{NO}_{2} 256.1338(\mathrm{M}+\mathrm{H})^{+}$, Found 256.1340.

Synthesis of Azabicycle C. A 100-mL round-bottom flask was charged with B $(610 \mathrm{mg}$, $2.39 \mathrm{mmol}), \mathrm{CH}_{2} \mathrm{Cl}_{2}(25 \mathrm{~mL})$, and $\mathrm{Ru}$ catalyst $\mathbf{D}(75 \mathrm{mg}, 0.12 \mathrm{mmol}, 5 \mathrm{~mol} \%)$ at $22{ }^{\circ} \mathrm{C}$. The reaction mixture was allowed to stir for $18 \mathrm{~h}$, after which time the volatiles were removed in vacuo. The resulting dark brown residue was purified by silica gel chromatography (dry load, 4:1 to 1:1 hexanes:EtOAc) to give $\mathbf{C}$ (323 $\mathrm{mg}, 1.42 \mathrm{mmol}$, 60\%) as slight brown oil. IR (neat): 3057 (w), 2976 (w), 2901 (w), 1716 (s), 1635 (s), 1412 (s). ${ }^{1} \mathrm{H} \mathrm{NMR}\left(400 \mathrm{MHz}, \mathrm{CDCl}_{3}\right)$ : reported as a 1:1 mixture of amide rotamers, $\delta$ 7.55-7.42 (m, 5H), 6.33 (br s, 1H), 6.21 (br s, 1H), 5.35 (br s, 1H), 4.74 (br s, 1H), 3.05-2.95 (m, 1H), 2.58-2.44 (m, 2H), 2.38-2.28 (m, 1H). ${ }^{13} \mathrm{C}$ NMR $(100 \mathrm{MHz}$, $\left.\mathrm{CDCl}_{3}\right): \delta 205.1,168.1,134.8,134.6,133.4,130.8,128.8,127.3,58.7,55.0,46.7,45.6$. HRMS ES $(\mathrm{m} / z)$ mass Calcd for $\mathrm{C}_{14} \mathrm{H}_{14} \mathrm{NO}_{2} 228.1025(\mathrm{M}+\mathrm{H})^{+}$, Found 228.1018.

Synthesis of Azabicyclic Precursor to 22. ${ }^{11}$ A 200-mL round-bottom flask was charged with C (1.35 g, $5.94 \mathrm{mmol})$, THF (59 $\mathrm{mL})$, and the reaction vessel was allowed to cool to $-78{ }^{\circ} \mathrm{C}$ in a dry ice/acetone bath. To this solution was added L-Selectride $(6.53 \mathrm{~mL}, 6.53$ $\mathrm{mmol}, 1.00 \mathrm{M}$ in THF) dropwise over a period of $10 \mathrm{~min}$, after which time the flask was removed from the dry ice batch and the mixture was allowed to stir at $22{ }^{\circ} \mathrm{C}$ for $1 \mathrm{~h}$. The reaction vessel was allowed to cool to $0^{\circ} \mathrm{C}$ in an ice-bath and the reaction was quenched by the sequential slow addition of a $1.0 \mathrm{M}$ solution of $\mathrm{NaOH}(20 \mathrm{~mL})$ and $\mathrm{H}_{2} \mathrm{O}_{2}(37 \mathrm{wt} \%$ in $\mathrm{H}_{2} \mathrm{O}, 20 \mathrm{~mL}$ ). At this point, the flask was removed from the ice-bath and the mixture was allowed to stir at $22{ }^{\circ} \mathrm{C}$ for $15 \mathrm{~min}$, after which time a $1.0 \mathrm{M}$ solution of $\mathrm{HCl}(20 \mathrm{~mL})$ was added. The resulting solution was washed with $\mathrm{CH}_{2} \mathrm{Cl}_{2}(3 \times 100 \mathrm{~mL})$. The combined organic layers were dried $\left(\mathrm{MgSO}_{4}\right)$, filtered, and the volatiles removed in vacuo. The remaining yellow residue was purified by silica gel chromatography (dry load, 2:1 to 1:2 hexanes:EtOAc) to deliver the precursor to 22 as colorless oil (1.09 $\mathrm{g}$,

(11) The precursor to $\mathbf{2 2}$ is the azabicycle containing a secondary alcohol derived from hydride reduction of the carbonyl in $\mathbf{C}$. The diastereomeric identity of this alcohol (endo vs. exo) was assigned by correlation to related azabicyclic substrates. See ref. 6. 
4.75 mmol, 80\%). IR (neat): 3408 (br), 2974 (w), 2945 (m), 2943 (m), 1621 (s) 1451 (s) 1067 (s), 696 (s). ${ }^{1} \mathrm{H}$ NMR (400 MHz, $\mathrm{CDCl}_{3}$, TMS): $\delta$ 7.50-7.39 (m, 5H), 6.51 (dd, $J=$ $5.9,2.0 \mathrm{~Hz}, 1 \mathrm{H}), 6.36(\mathrm{dd}, J=5.9,2.0 \mathrm{~Hz}, 1 \mathrm{H}), 5.14$ (br s, $1 \mathrm{H}), 4.47$ (br s, $1 \mathrm{H}$ ), 4.05-4.01 (m, 1H), 2.50-2.44 (m, 1H), $2.24(\mathrm{~d}, J=9.7 \mathrm{~Hz}, 1 \mathrm{H}), 2.14-2.08(\mathrm{~m}, 1 \mathrm{H}), 1.91$ (d, $J=14.8 \mathrm{~Hz}, 1 \mathrm{H}), 1.79(\mathrm{~d}, J=14.8 \mathrm{~Hz}, 1 \mathrm{H}) .{ }^{13} \mathrm{C} \mathrm{NMR}\left(100 \mathrm{MHz}, \mathrm{CDCl}_{3}\right): \delta 167.3$, $136.7,135.6,133.2,130.3,128.6,127.3,65.7,60.1,55.8,37.3,35.5$. HRMS ES $(m / z)$ mass Calcd for $\mathrm{C}_{14} \mathrm{H}_{15} \mathrm{NO}_{2} 229.1098(\mathrm{M})^{+}$, Found 229.1102 .

Synthesis of Olefin Methathesis Substrate 22. A 200-mL round-bottom flask, fitted with an addition funnel, was charged with the azabicyclic precursor to $22(1.09 \mathrm{~g}, 4.75$ $\mathrm{mmol})$, THF $(48 \mathrm{~mL})$, and the mixture was allowed to cool to $-78{ }^{\circ} \mathrm{C}$ in a dry ice/acetone bath. To this solution was added DIBAl-H $(16.9 \mathrm{~mL}, 23.8 \mathrm{mmol}, 20 \%$ by volume in hexane) dropwise over a period of $15 \mathrm{~min}$. The resulting mixture was allowed to stir at $78^{\circ} \mathrm{C}$ for $10 \mathrm{~min}$, after which time the flask was removed from the dry ice bath and the mixture was allowed to stir at $22{ }^{\circ} \mathrm{C}$ for $2 \mathrm{~h}$. At this time, the mixture was allowed to cool to $0{ }^{\circ} \mathrm{C}$ in an ice-bath and the reaction was quenched by the addition of a saturated aqueous solution of potassium sodium tartrate $(20 \mathrm{~mL})$. At this point, the flask was removed from the ice-bath and the mixture was allowed to stir at $22{ }^{\circ} \mathrm{C}$ for $15 \mathrm{~h}$, after which time it was washed with EtOAc $(3 \times 100 \mathrm{~mL})$, dried $\left(\mathrm{Na}_{2} \mathrm{SO}_{4}\right)$, filtered, and the volatiles removed in vacuo. The dark brown residue was purified by silica gel chromatography $\left(\mathrm{CH}_{2} \mathrm{Cl}_{2}\right.$ to $\left.94: 5: 1 \mathrm{CH}_{2} \mathrm{Cl}_{2}: \mathrm{MeOH}: \mathrm{NEt}_{3}\right)$ to provide 22 as slight yellow solid (510 mg, $2.37 \mathrm{mmol}, 50 \%$ ). mp: 102-104 ${ }^{\circ} \mathrm{C}$. IR (neat): 3408 (br), 2930 (s), 2836 (w), 1489 (m) 1438 (m), 1042 (s). ${ }^{1} \mathrm{H}$ NMR (400 MHz, $\left.\mathrm{CDCl}_{3}, \mathrm{TMS}\right): \delta$ 7.26-7.21 (m, $5 \mathrm{H}), 6.29(\mathrm{~s}, 2 \mathrm{H}), 3.90-3.85(\mathrm{~m}, 1 \mathrm{H}), 3.53(\mathrm{~s}, 2 \mathrm{H}), 3.50$ (br s, 2H), 2.28 (br s, $1 \mathrm{H}), 2.24$ $(\mathrm{dd}, J=6.0,3.7 \mathrm{~Hz}, 1 \mathrm{H}), 2.20(\mathrm{dd}, J=6.0,3.7 \mathrm{~Hz}, 1 \mathrm{H}), 1.81-1.80(\mathrm{~m}, 1 \mathrm{H}), 1.78-1.77$ $(\mathrm{m}, 1 \mathrm{H}) .{ }^{13} \mathrm{C}$ NMR $\left(100 \mathrm{MHz}, \mathrm{CDCl}_{3}\right): \delta 139.9,135.3,128.6,128.3,126.8,66.1,64.0$, 57.8, 37.8. HRMS ES $(\mathrm{m} / \mathrm{z})$ mass Calcd for $\mathrm{C}_{14} \mathrm{H}_{18} \mathrm{NO} 216.1388(\mathrm{M}+\mathrm{H})^{+}$, Found 216.1399 .

Synthesis of Olefin Metathesis Substrate 20. ${ }^{12}$ Azabicycle 20 was prepared by TBS protection of the alcohol in 22. IR (neat): 2925 (s), 2853 (w), 1252 (w), 1065 (s). ${ }^{1} \mathrm{H}$ NMR (400 MHz, CDCl $): \delta 7.38(\mathrm{~d}, J=7.2 \mathrm{~Hz}, 2 \mathrm{H}), 7.31(\mathrm{t}, J=7.2 \mathrm{~Hz}, 1 \mathrm{H}), 7.24(\mathrm{~d}, J=$ $7.2 \mathrm{~Hz}, 2 \mathrm{H}), 5.98(\mathrm{br} \mathrm{s}, 2 \mathrm{H}), 4.00(\mathrm{dd}, J=5.2 \mathrm{~Hz}, 1 \mathrm{H}), 3.60(\mathrm{br} \mathrm{s}, 2 \mathrm{H}), 3.46(\mathrm{br} \mathrm{s}, 2 \mathrm{H})$, 2.14 (br s, 2H), 1.56 (d, $J=12.0,2 \mathrm{H}), 0.85(\mathrm{~s}, 9 \mathrm{H}),-0.44$ (s, 6H). ${ }^{13} \mathrm{C} \mathrm{NMR}(100 \mathrm{MHz}$, $\left.\mathrm{CDCl}_{3}\right): \delta 133.7,132.6,128.8,126.8,65.2,64.1,57.7,37.5,25.9,17.9,0.02,-4.7$. HRMS ES $(\mathrm{m} / \mathrm{z})$ mass Calcd for $\mathrm{C}_{20} \mathrm{H}_{31} \mathrm{NOSi} 329.2184(\mathrm{M})^{+}$, Found 329.2175.

(12) For the TBS protection of a related azabicycle see ref. 6. 
Cortez, et al.; Page S17
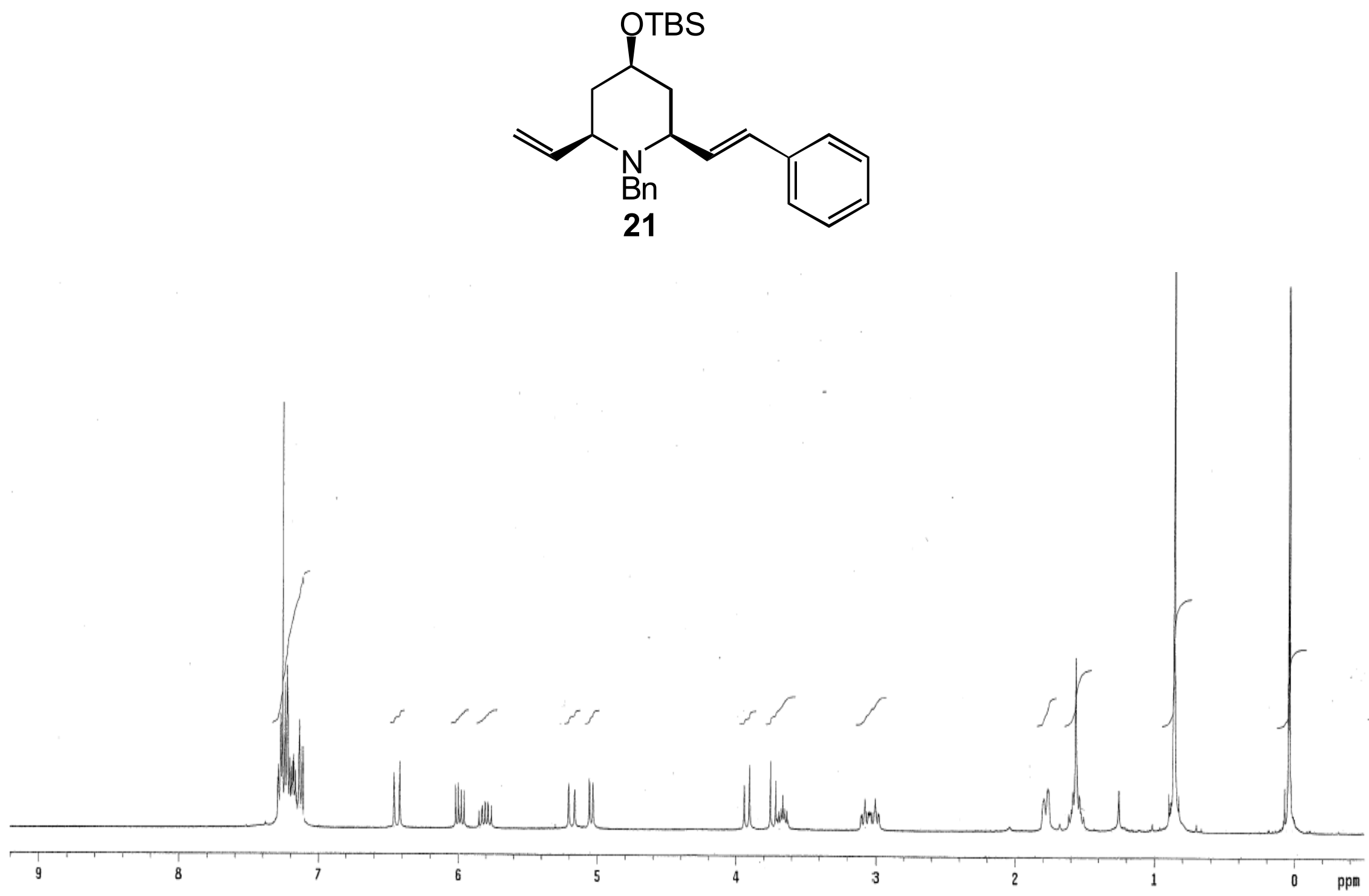

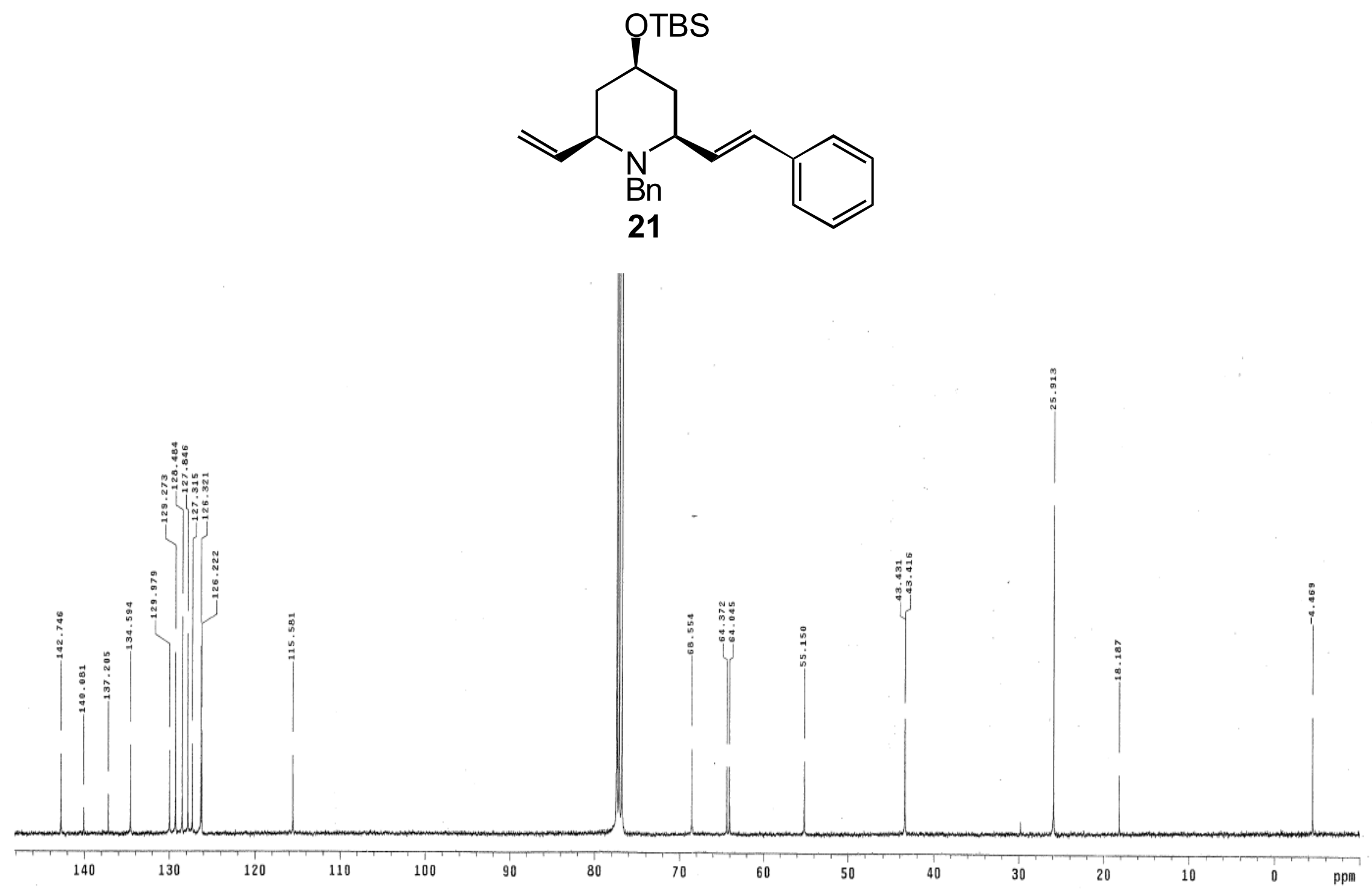

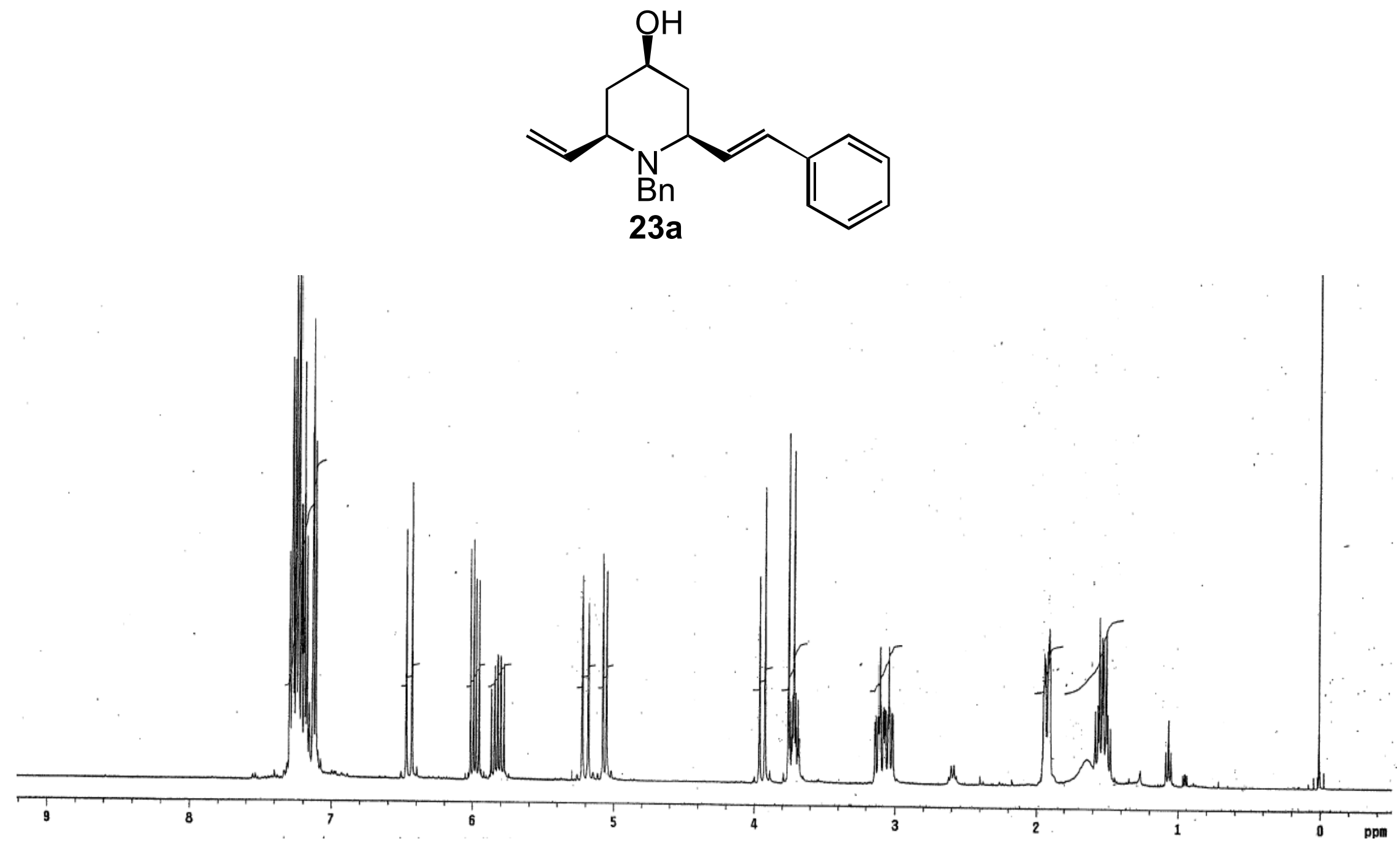

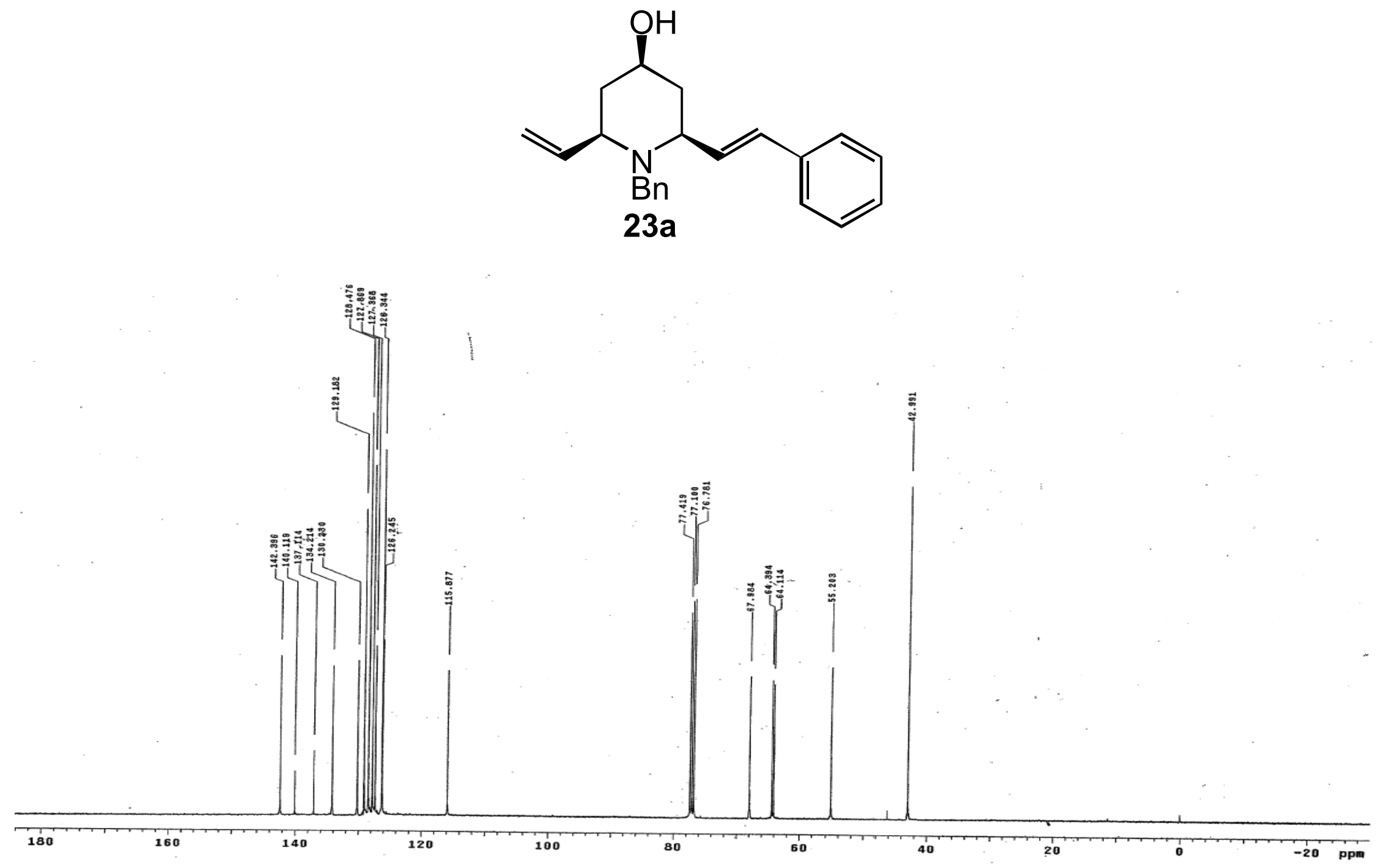


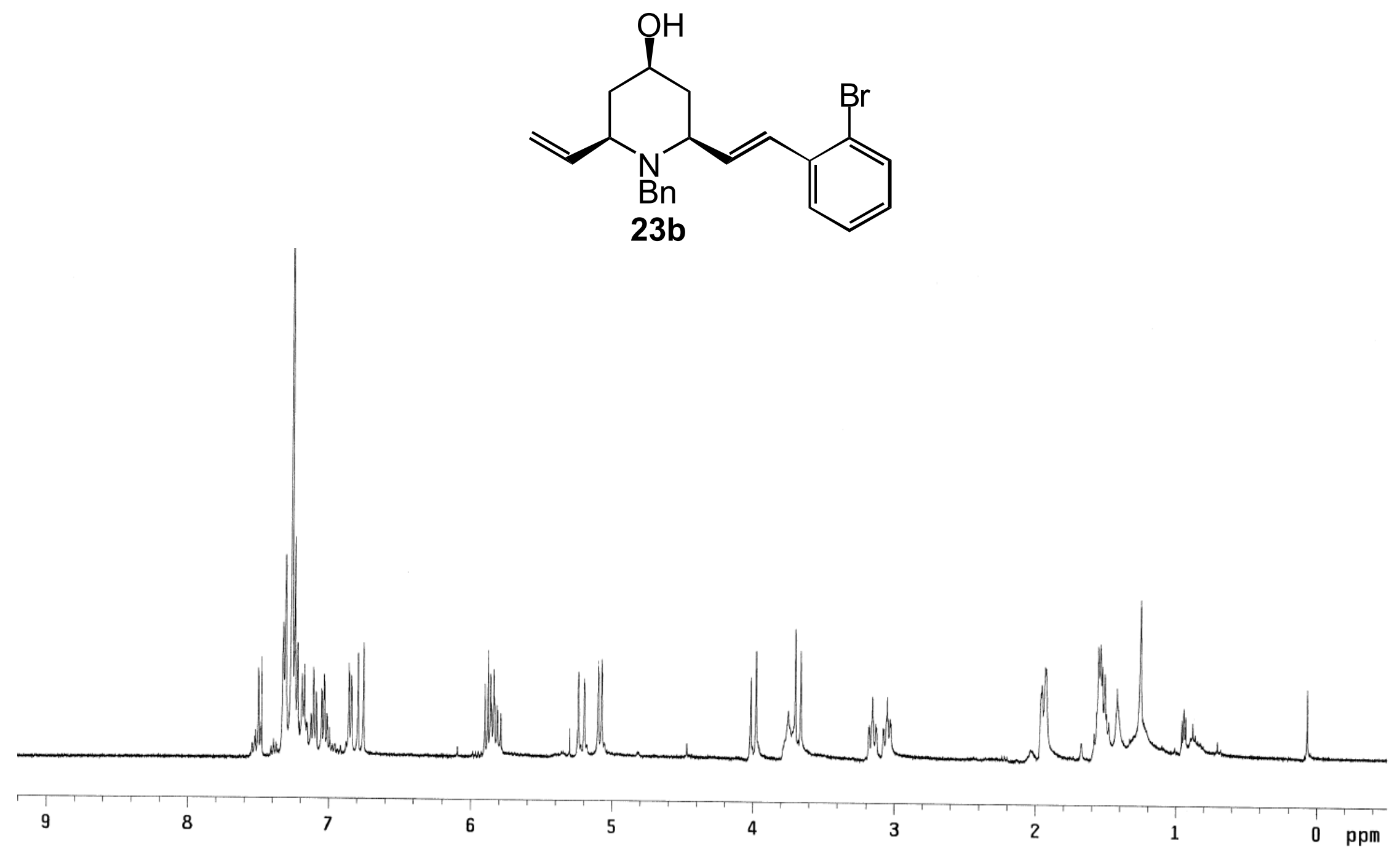



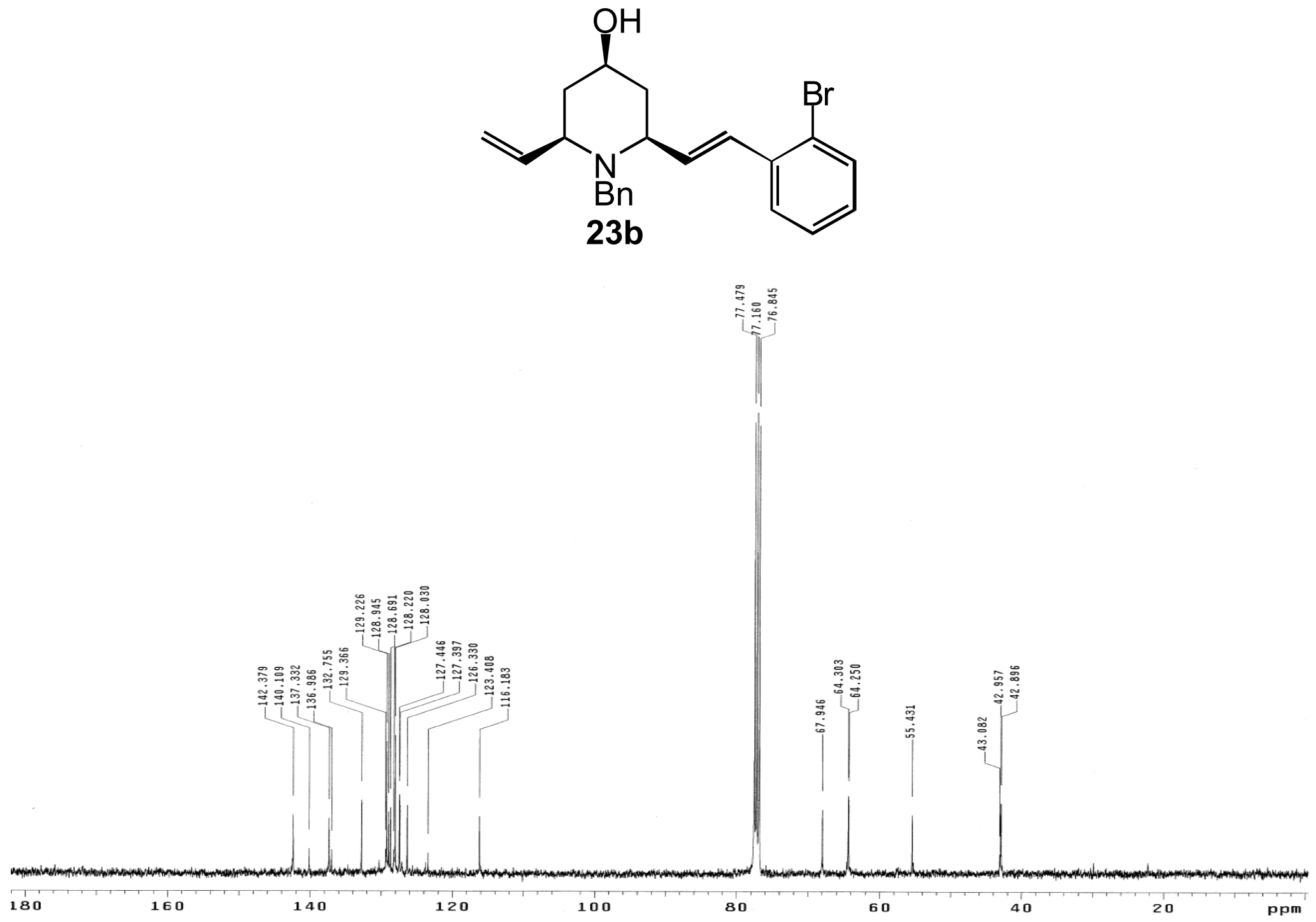

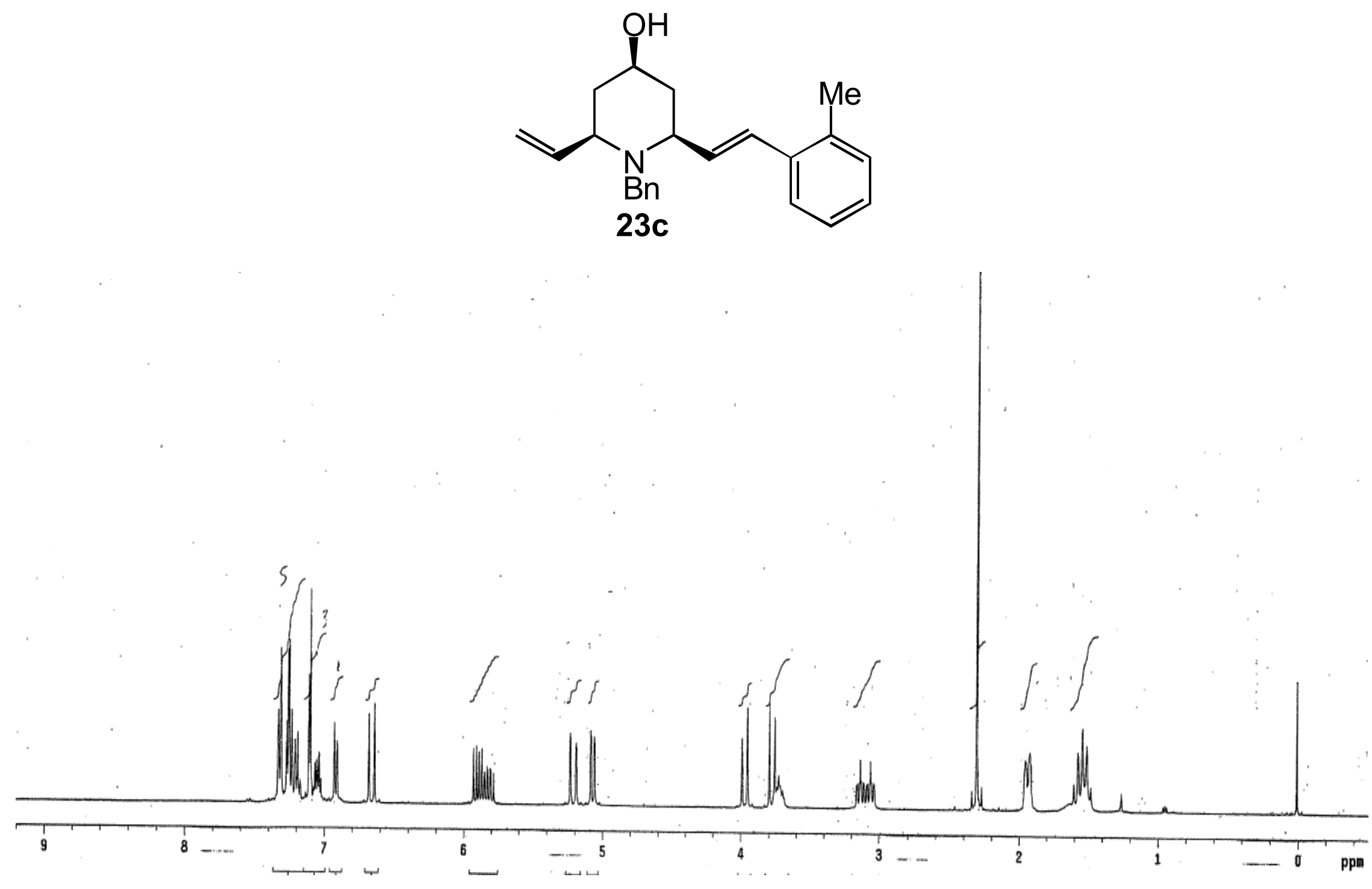

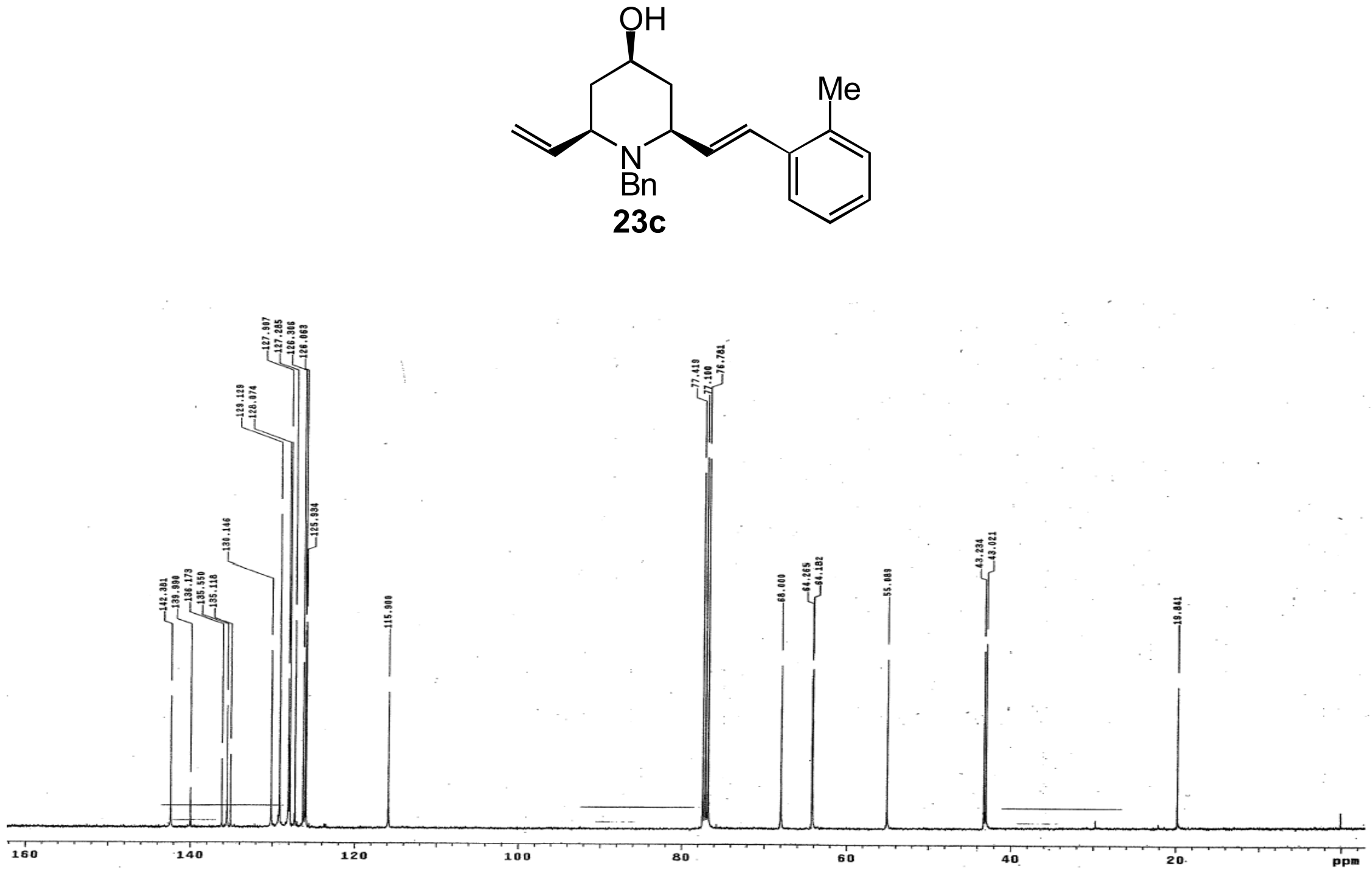


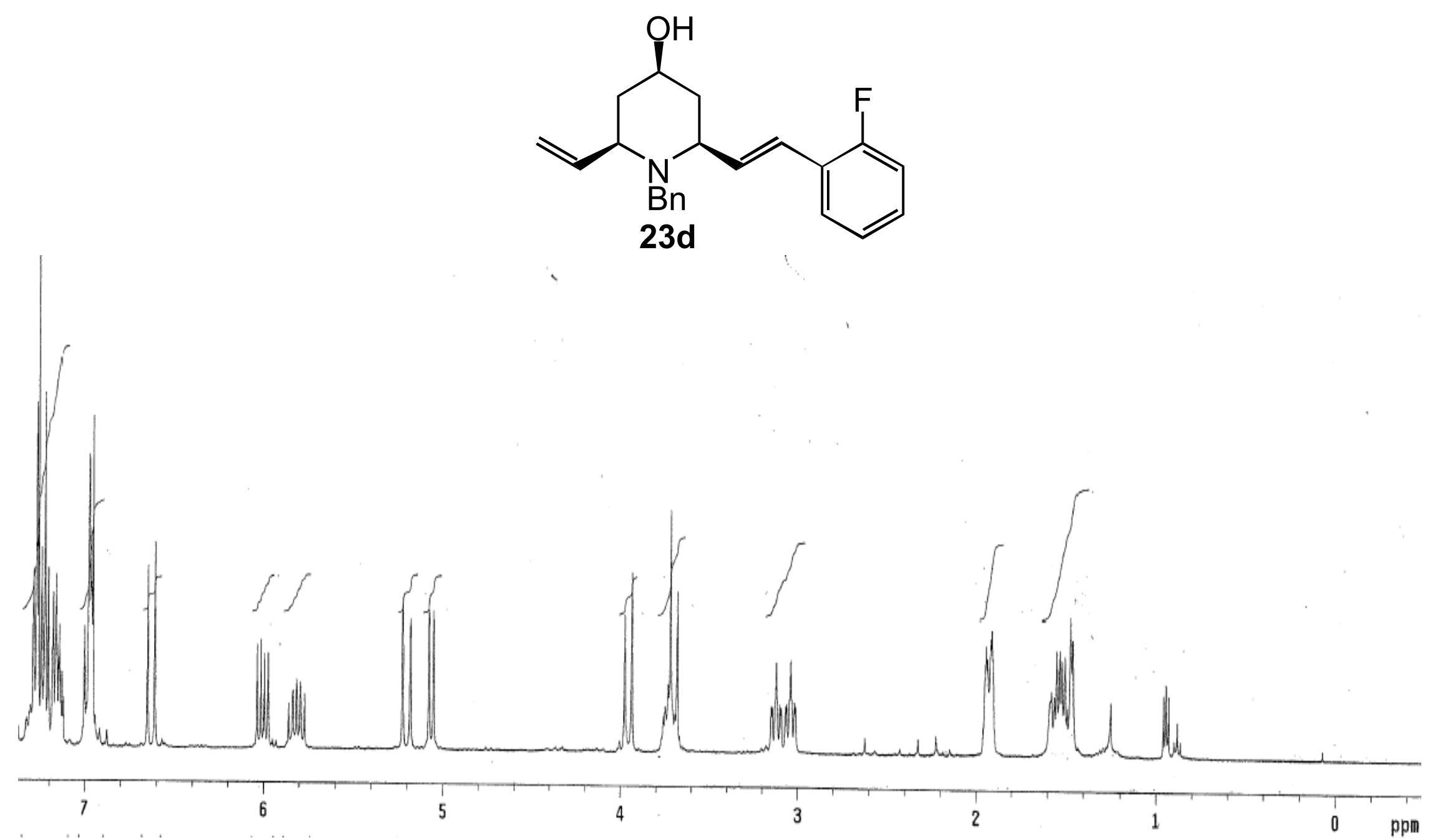




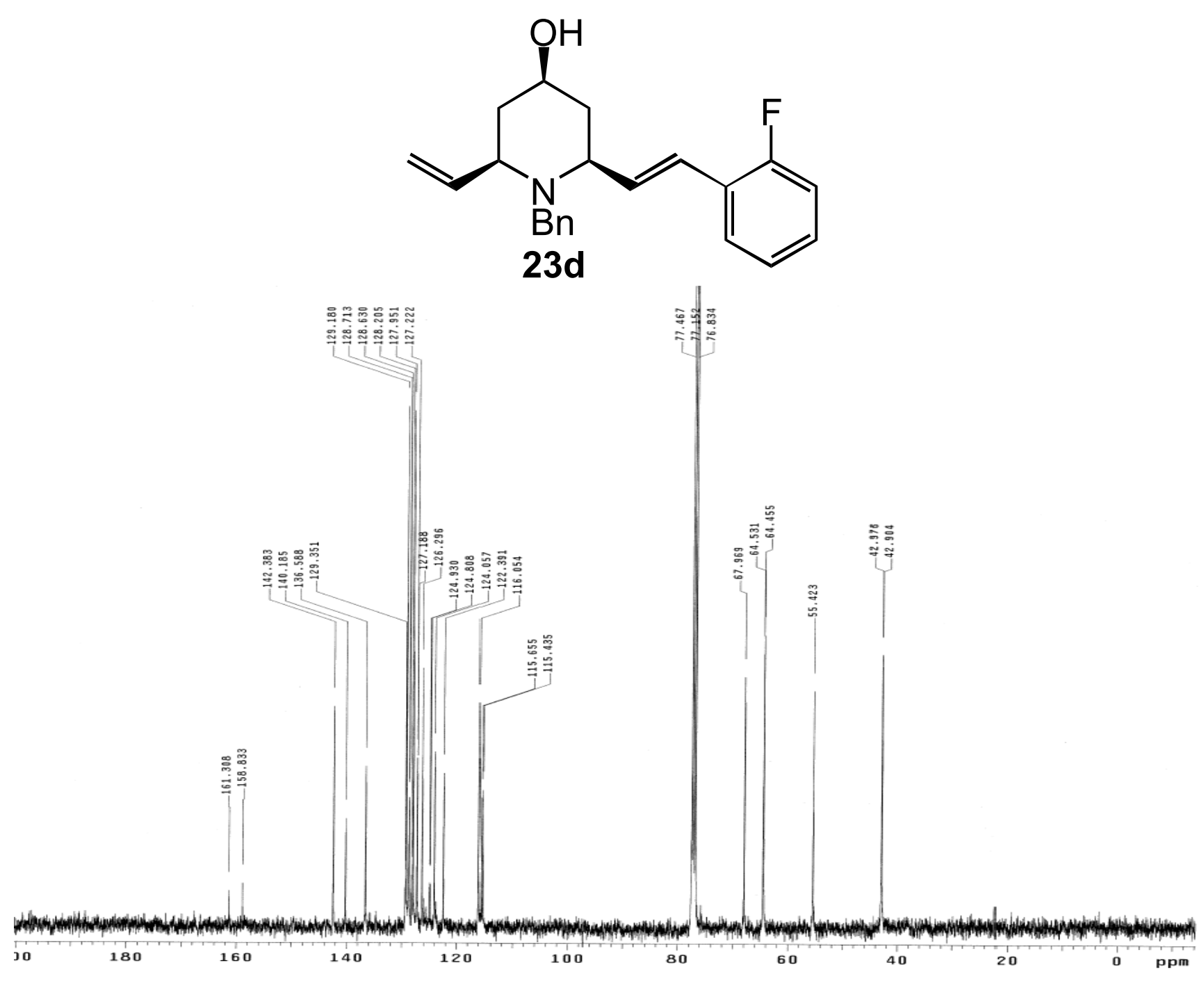




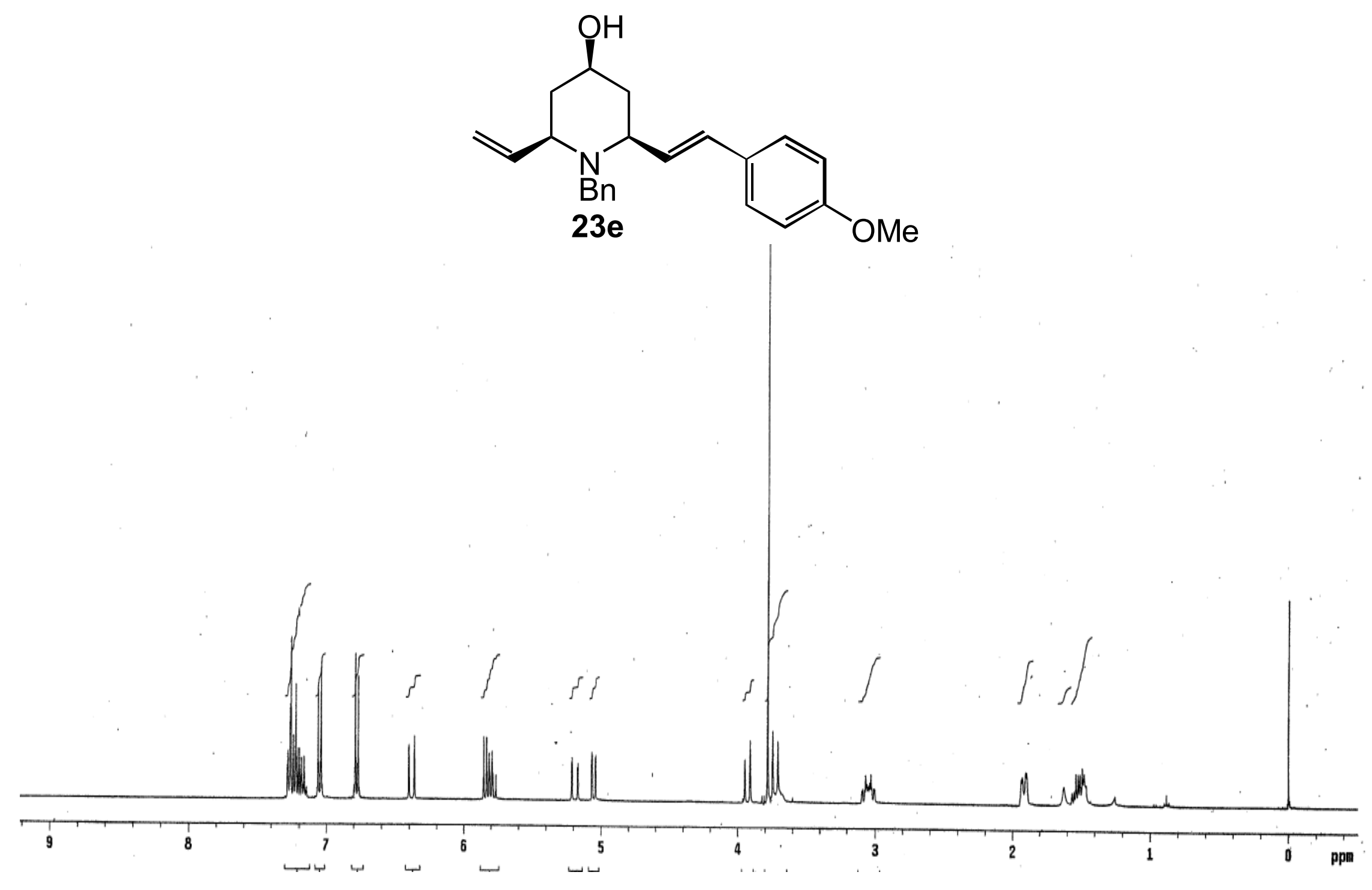



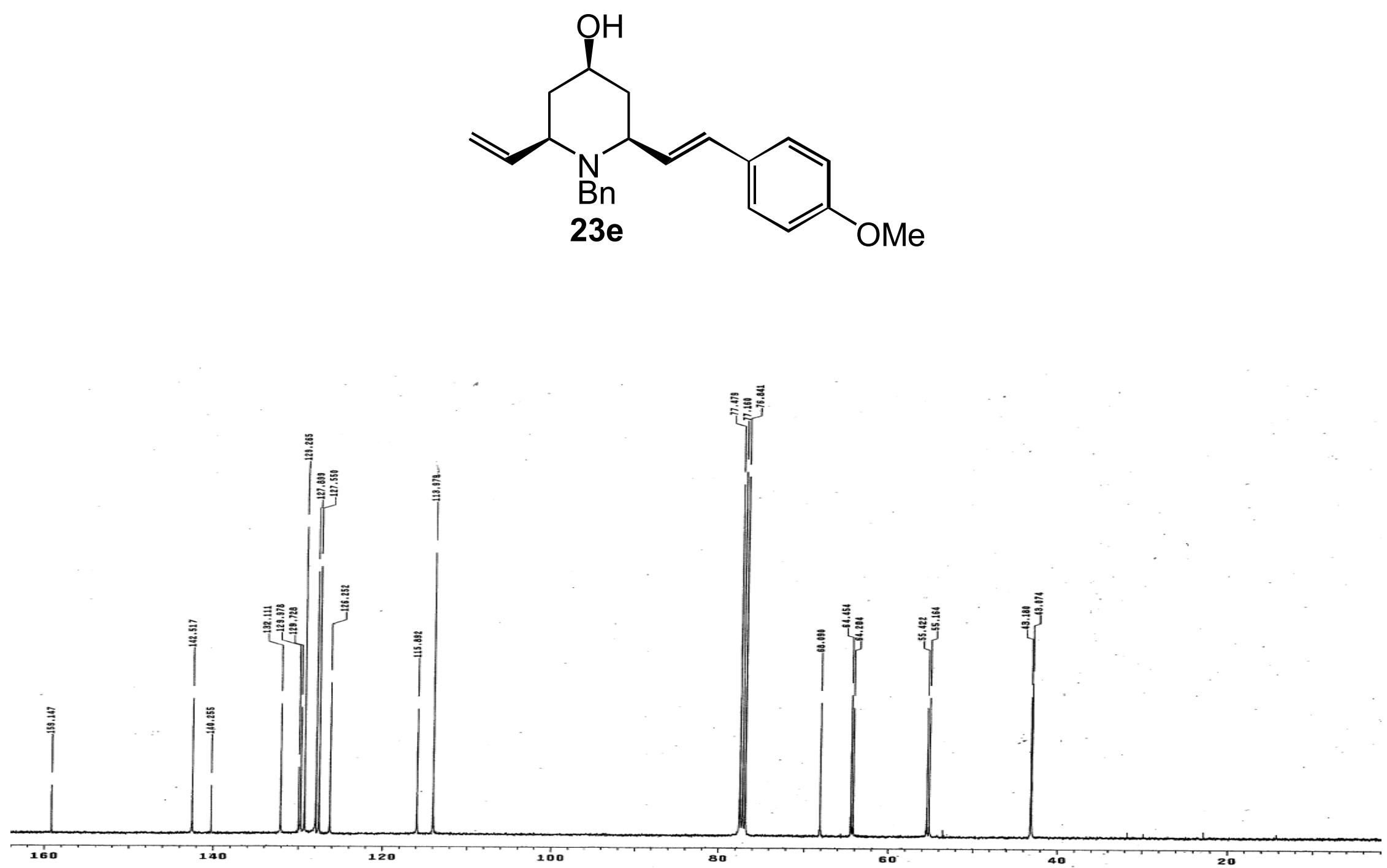

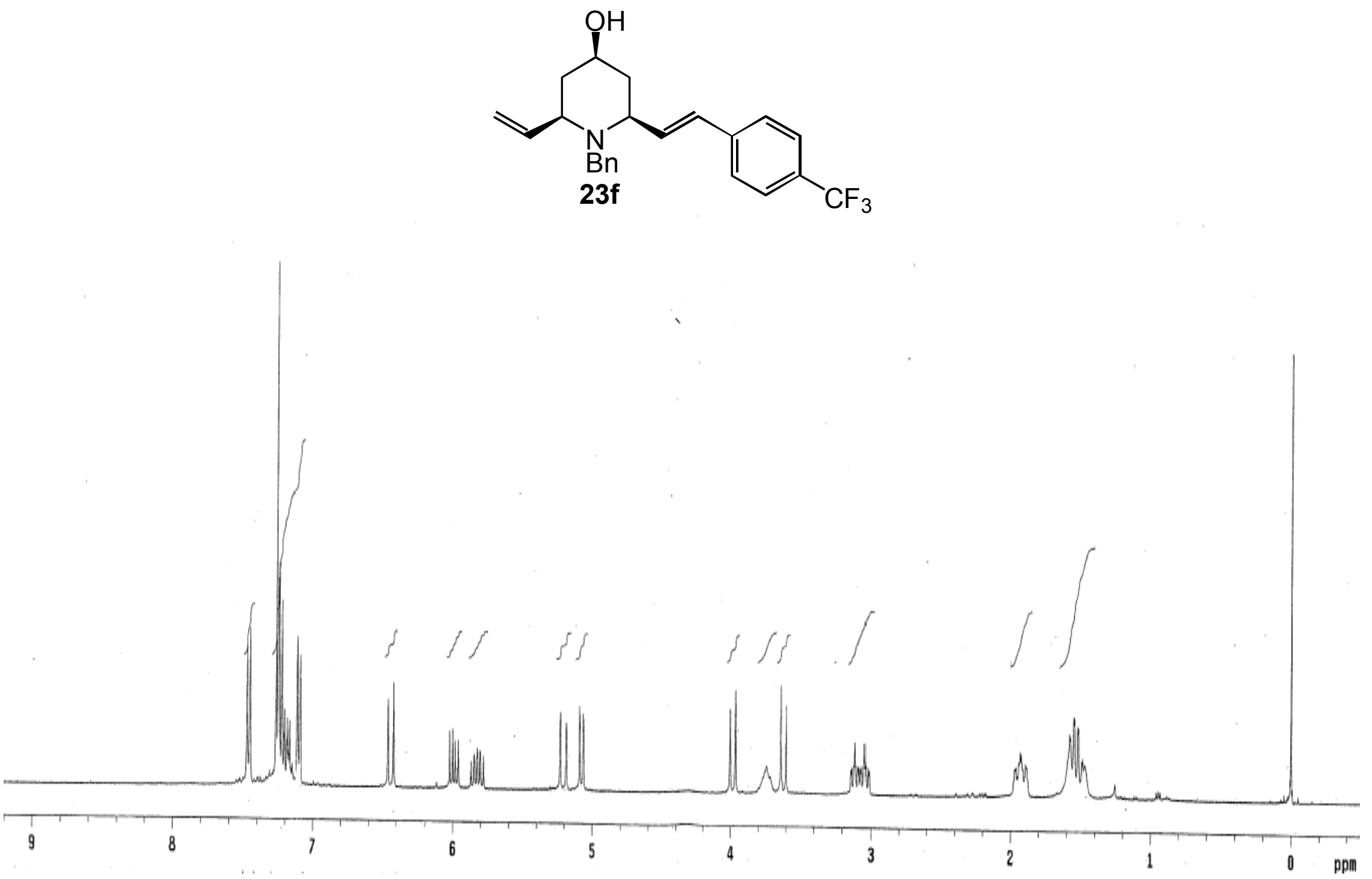

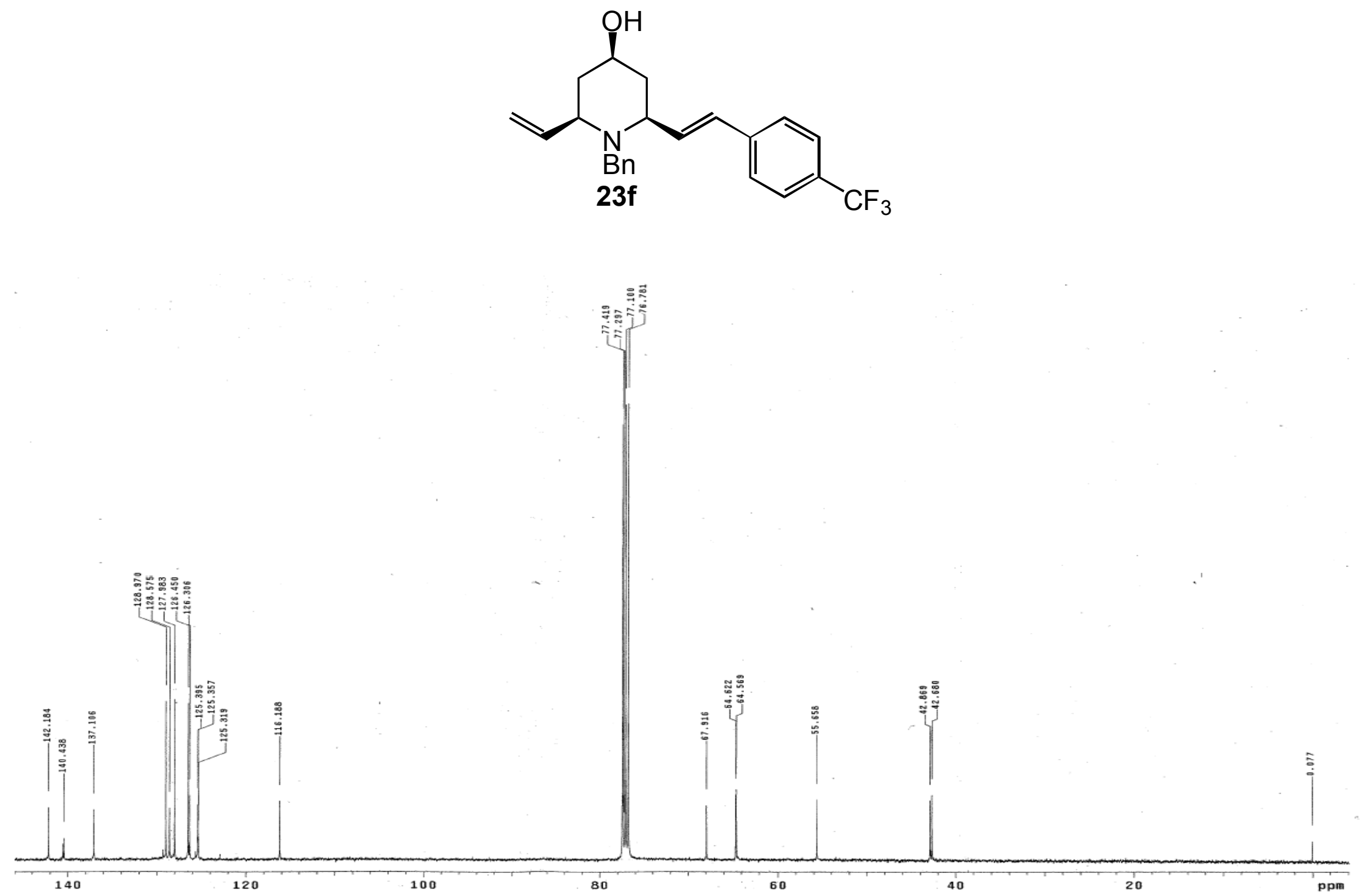

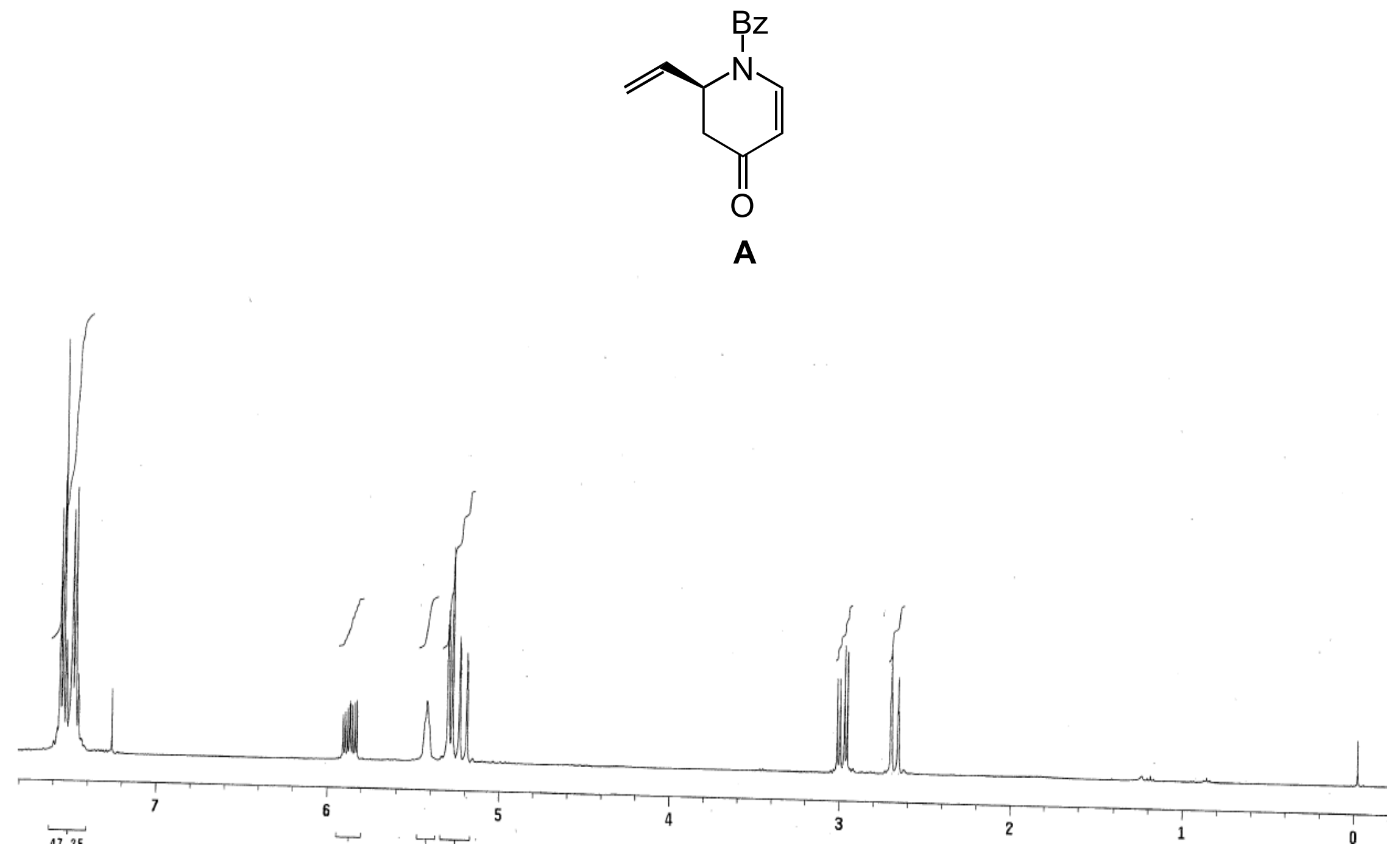


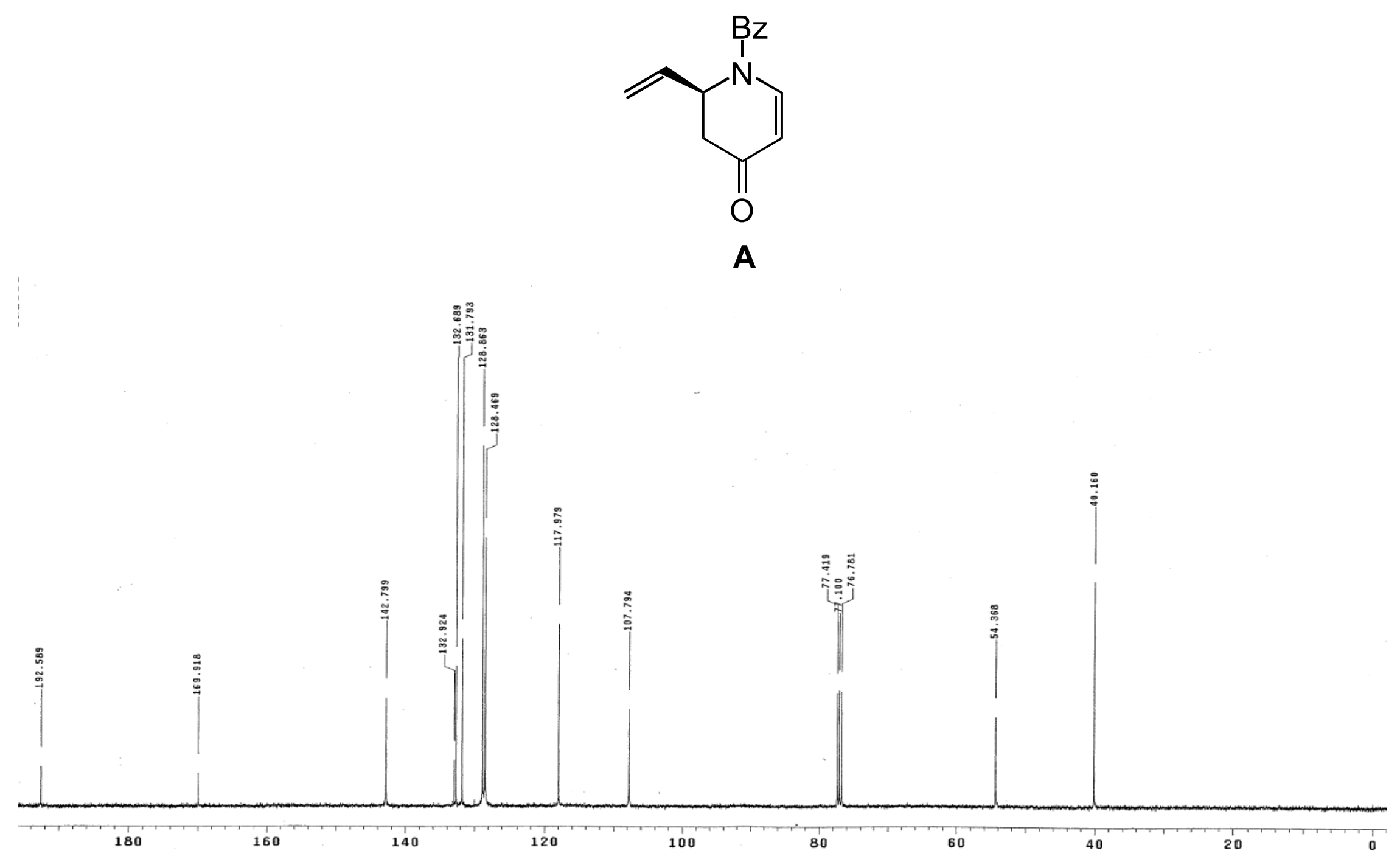



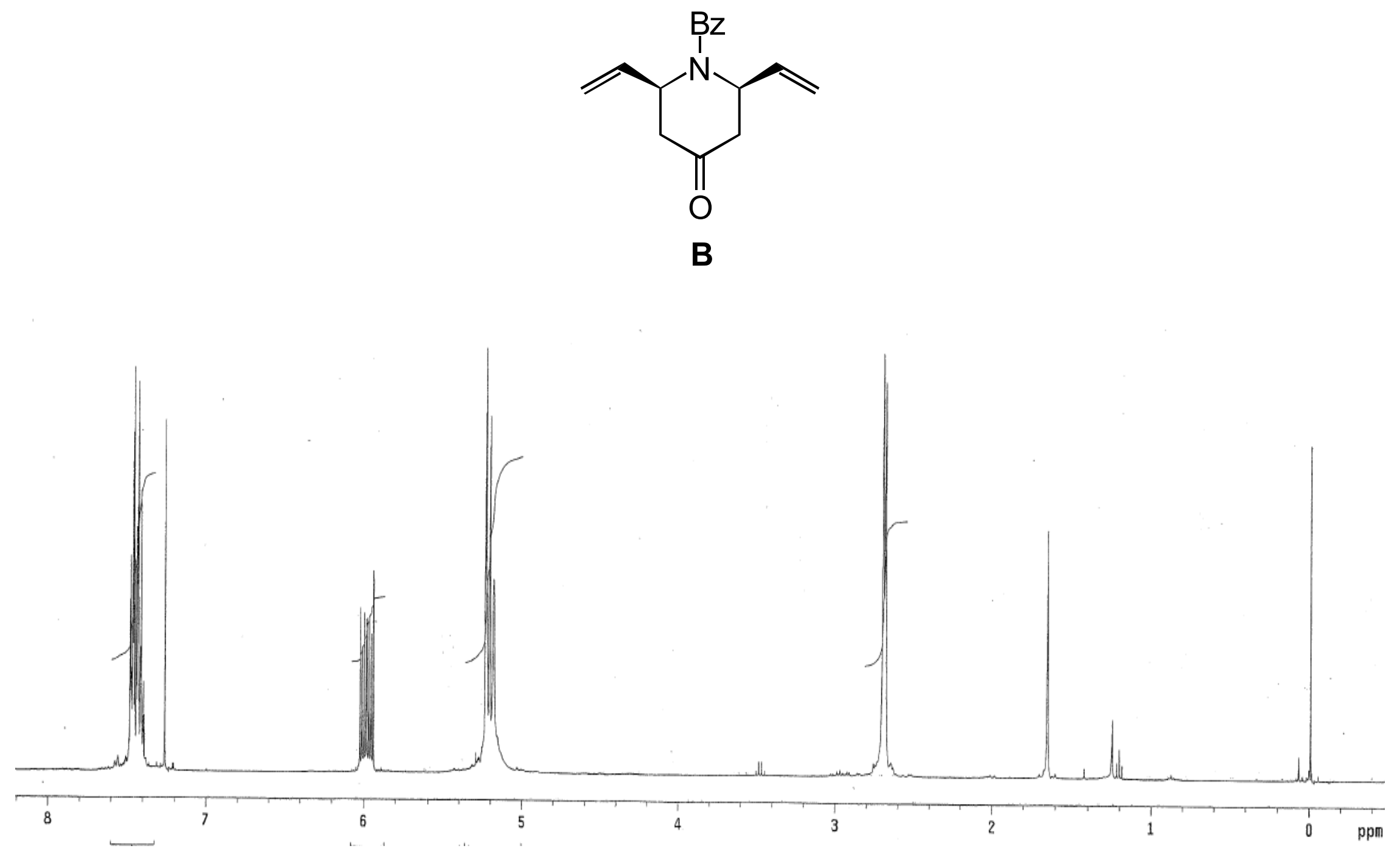


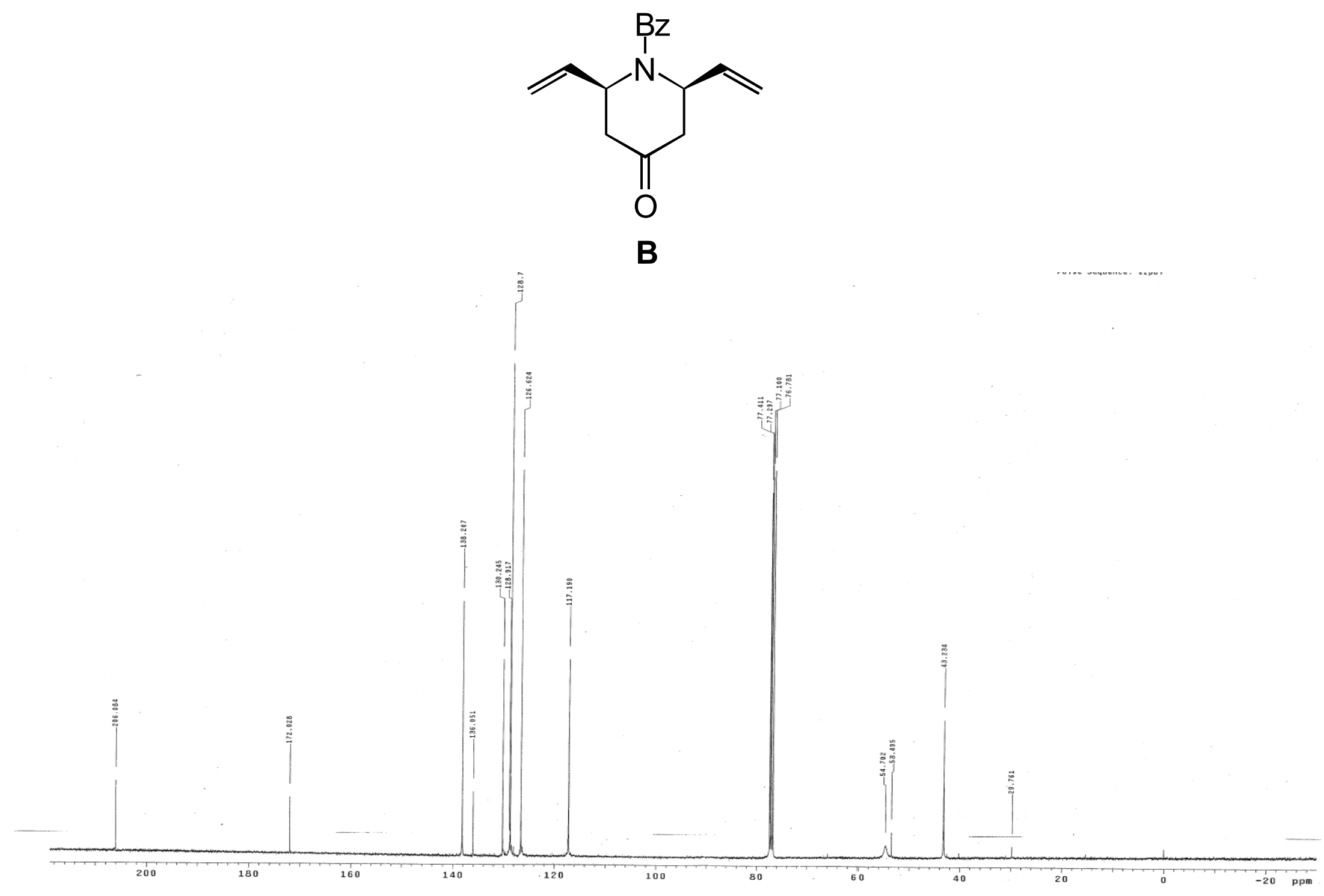



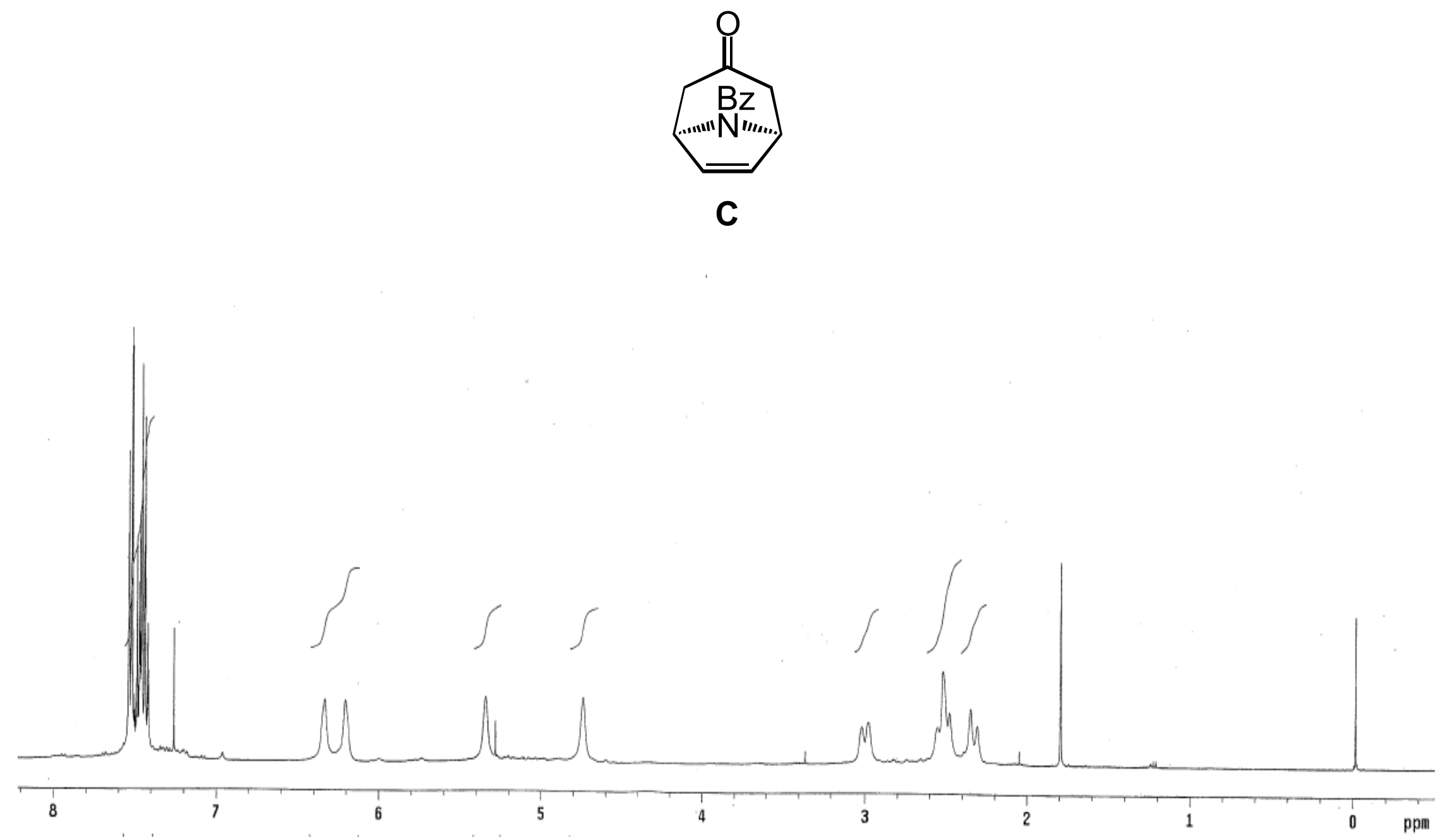


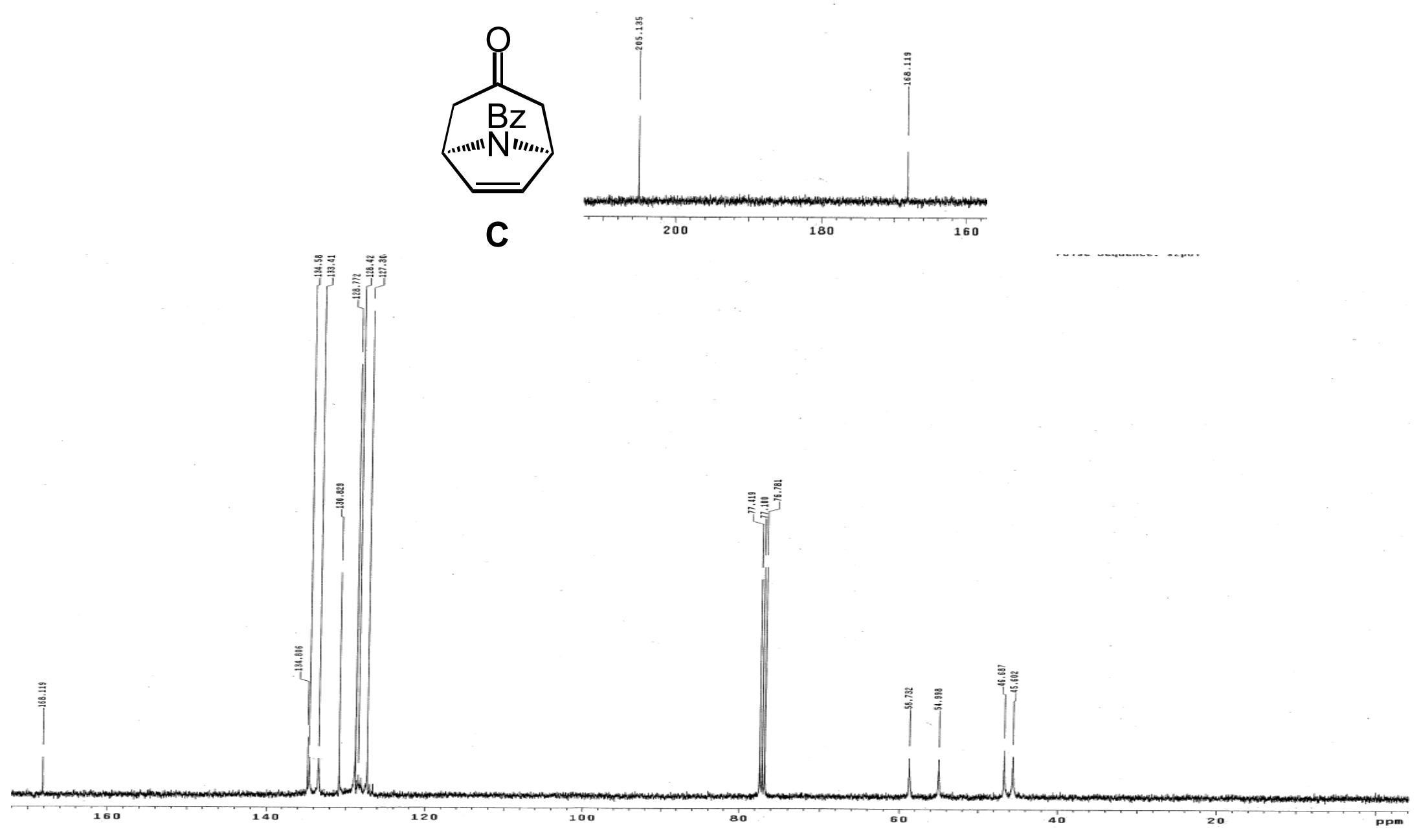




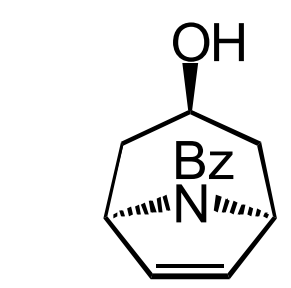

precursor to 22

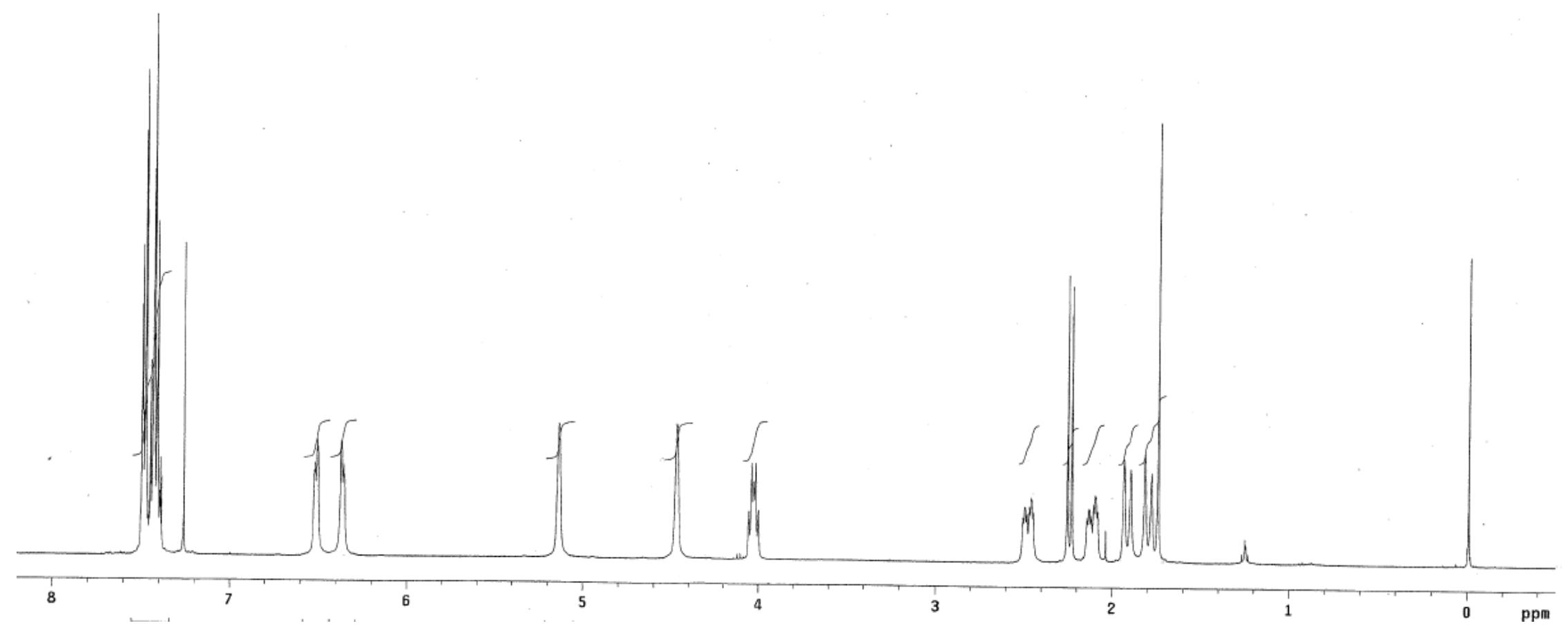




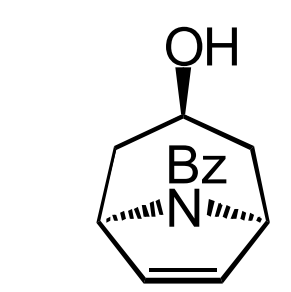

precursor to 22

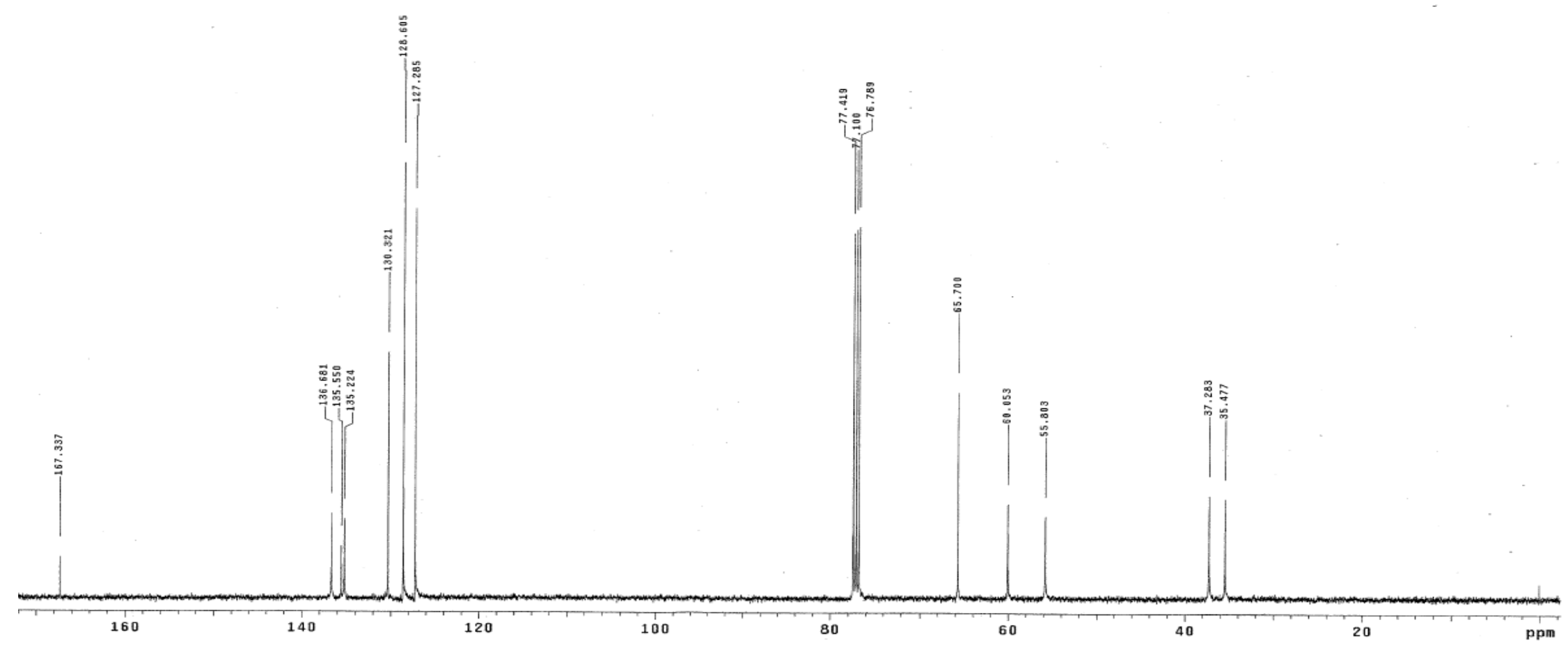



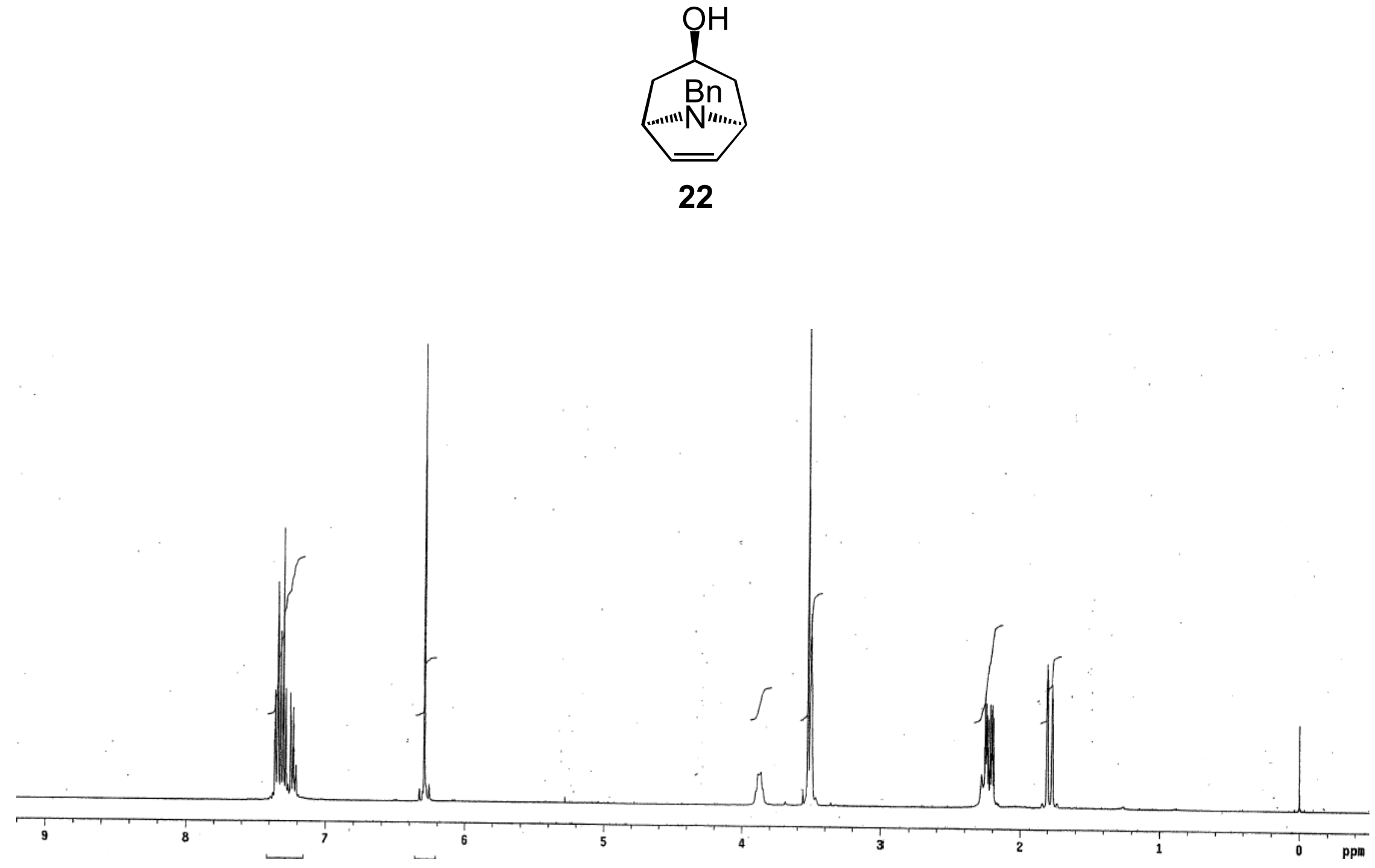


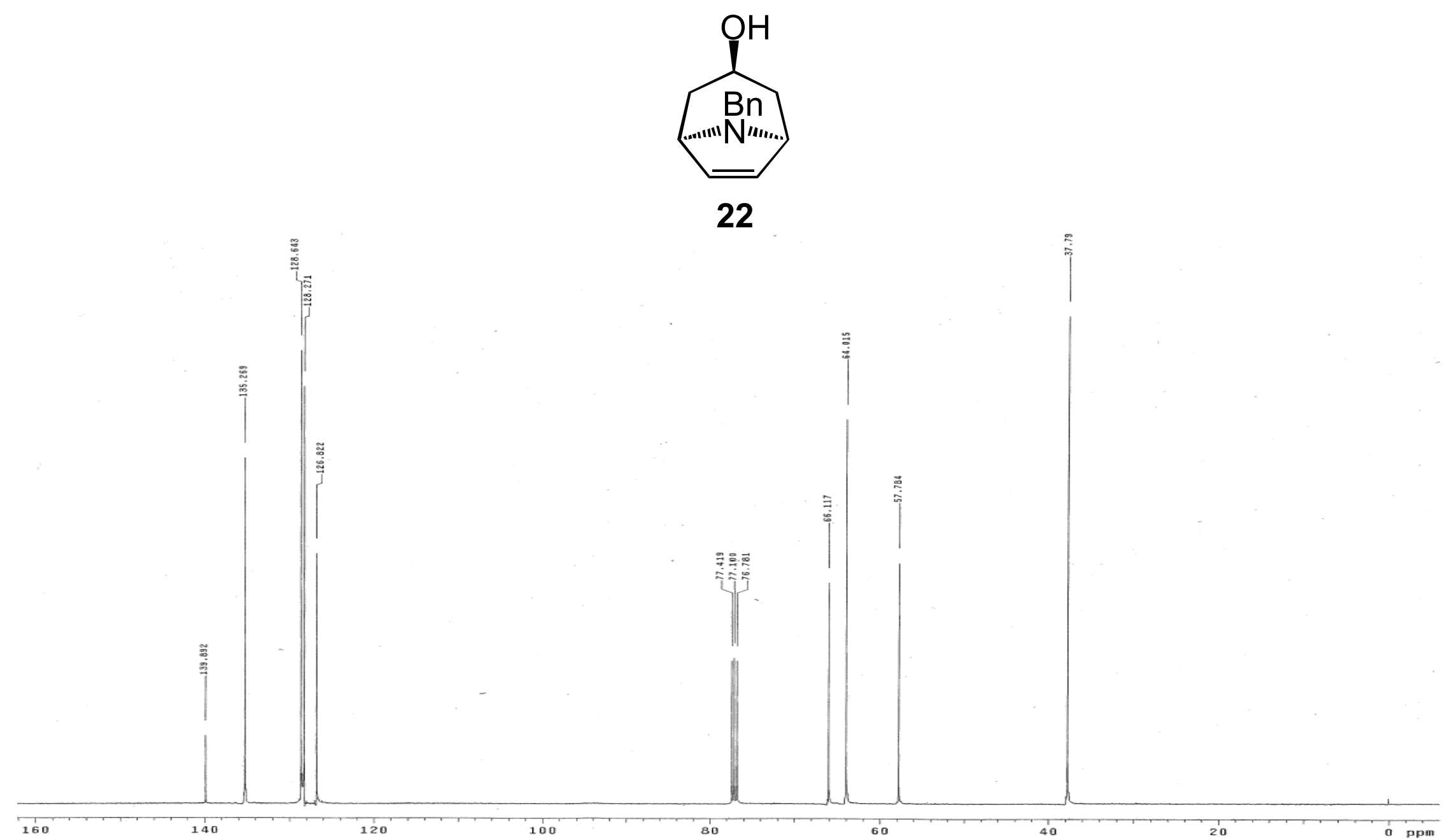



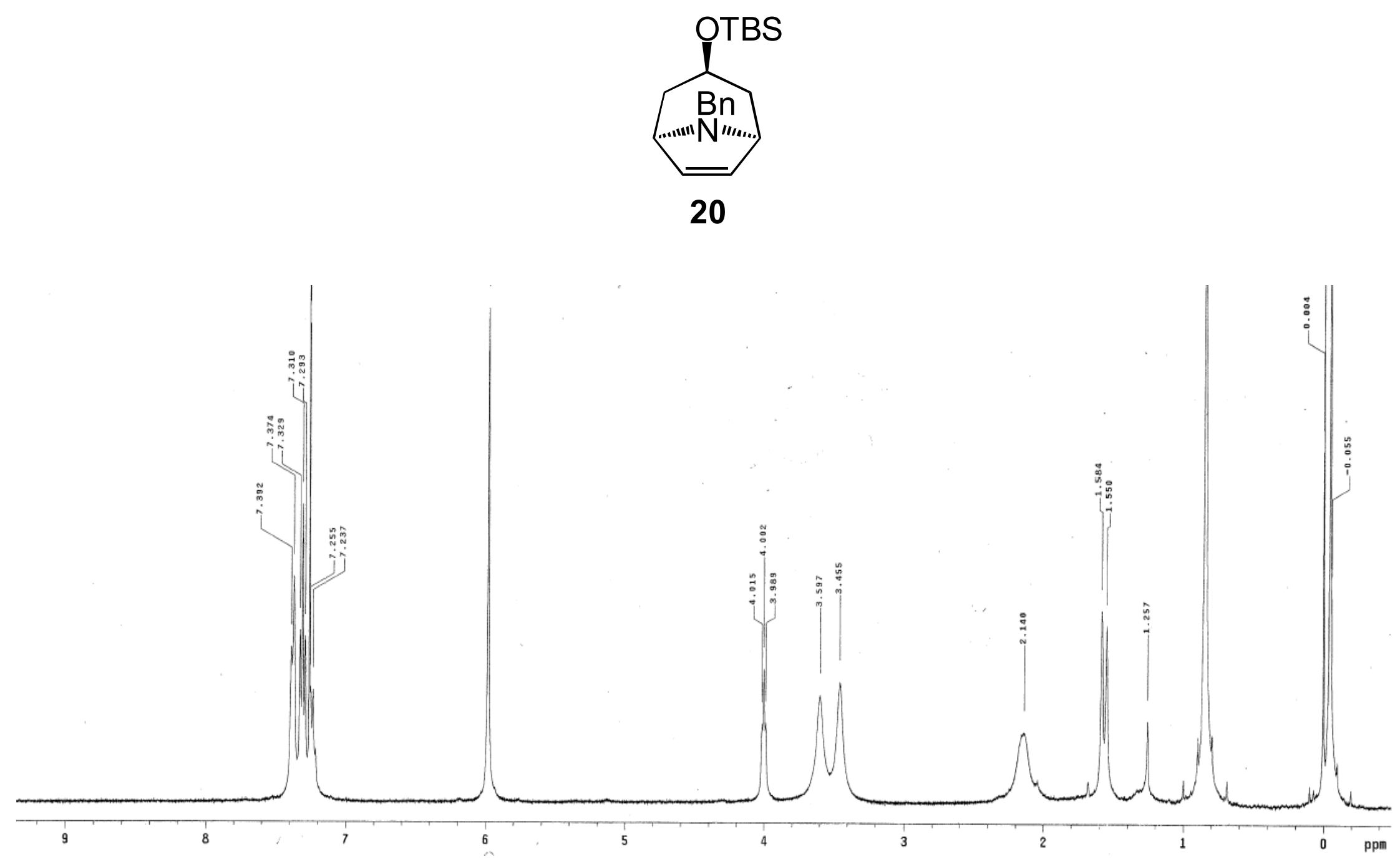


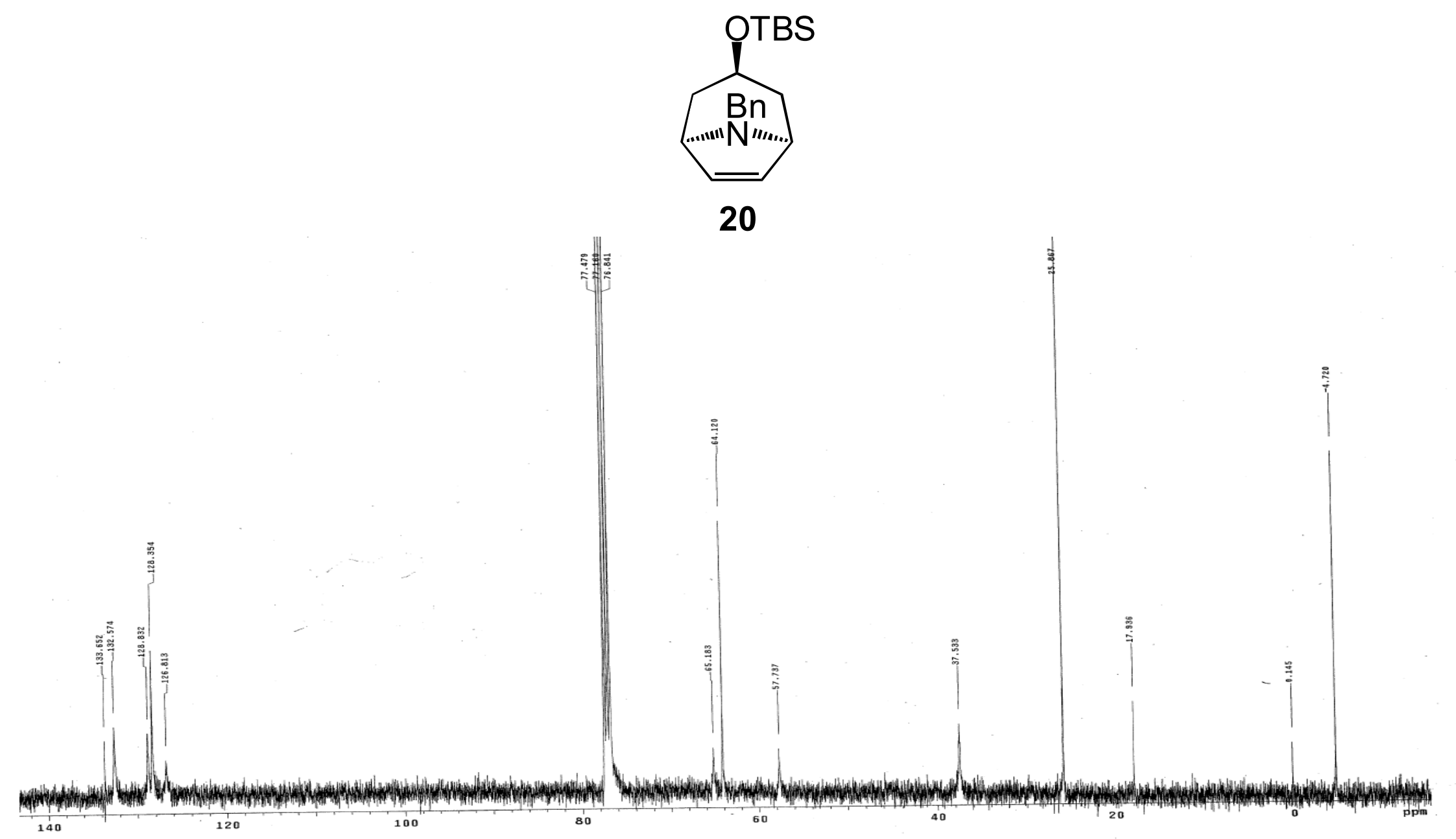

